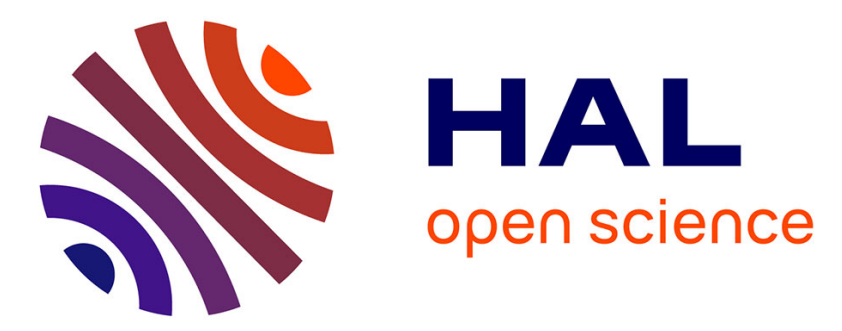

\title{
Multiscale empirical mode decomposition of density fluctuation images very near above and below the critical point of SF6
}

Ana Oprisan, Yves Garrabos, Carole Lecoutre-Chabot, Daniel Beysens

\section{To cite this version:}

Ana Oprisan, Yves Garrabos, Carole Lecoutre-Chabot, Daniel Beysens. Multiscale empirical mode decomposition of density fluctuation images very near above and below the critical point of SF6. Physica A: Statistical Mechanics and its Applications, 2021, 561, 125293 (18 p.). 10.1016/j.physa.2020.125293 . hal-02947750

\author{
HAL Id: hal-02947750 \\ https://hal.science/hal-02947750
}

Submitted on 5 Oct 2020

HAL is a multi-disciplinary open access archive for the deposit and dissemination of scientific research documents, whether they are published or not. The documents may come from teaching and research institutions in France or abroad, or from public or private research centers.
L'archive ouverte pluridisciplinaire HAL, est destinée au dépôt et à la diffusion de documents scientifiques de niveau recherche, publiés ou non, émanant des établissements d'enseignement et de recherche français ou étrangers, des laboratoires publics ou privés. 


\title{
Multiscale empirical mode decomposition of density fluctuation images very near above and below the critical point of $\mathrm{SF}_{6}$
}

\author{
Ana Oprisan* \\ Department of Physics and Astronomy, College of Charleston, 66 George Street, \\ Charleston, SC 29424 \\ Yves Garrabos, Carole Lecoutre-Chabot \\ CNRS, Université de Bordeaux, Bordeaux INP, ICMCB, UMR 5026, F-33600 Pessac, \\ France \\ Daniel Beysens \\ Physique et Mécanique des Milieux Hétérogènes, CNRS, ESPCI, PSL Research University, \\ Sorbonne Universitée, Sorbonne Paris Citée, Paris, France
}

\begin{abstract}
We use a multiscale approach to investigate the dynamics of fluctuations near the critical point of sulfur hexafluoride $\left(\mathrm{SF}_{6}\right)$ in microgravity. Rather than increasing the fitting model's complexity during the critical temperature crossing, we used a different approach to finding the thermal diffusivity coefficient (above critical temperature), which can then be distinguished from an effective diffusion coefficient (below critical temperature). We first separate different spatial scales from the original images using the Bidimensional Empirical Mode Decomposition (BEMD) technique. The spatial scale represented by an Intrinsic Mode Function (IMF) image was analyzed using the Dynamic Differential Method (DDM). The Intermediate Scattering Function (ISF) of each IMF was used for computing the structure factor and the relaxation time of fluctuations. We found that the first IMF returns over $90 \%$ of the spatial and temporal knowledge contained in the original image, providing thus thermal diffusivity
\end{abstract}

\footnotetext{
* Corresponding author

Email address: oprisana@cofc.edu (Ana Oprisan)
} 
coefficient above the critical temperature and effective diffusion coefficients below the critical temperature very close in magnitude. The relaxation time associated with the distinguishable structures observed in the second IMF could be attributed to the fractal nature of fluctuations. and to light scattering at low wavenumber during the stationary behavior and the transient evolution of the critical fluid cell, which are not easy to detect in the original image. The third order IMF presents no noticeable structure, and the associated relaxation time is not physically significant.

Keywords: sulfur hexafluoride, microgravity, critical fluctuations, relaxation time, diffusivity, effective diffusion coefficient

2010 MSC: 00-01, 99-00

\section{Introduction}

Dynamic Differential Microscopy (DDM) is an appealing experimental technique to extract the relaxation time of dynamical phenomena $[1,2,3,4]$. It has recently been applied to critical density fluctuations from light scattering im-

5 ages of systems approaching the liquid-gas critical point of pure fluids from the homogeneous domain [5]. In such experiments, the image processing performed with the dynamic structure factor (DSF) algorithm produces results consistent with the modern theory of critical phenomena. One prediction is that such systems are characterized by only one spatial scale of critical density fluctuations related to their characteristic size, the critical correlation length, and a single critical relaxation time of density fluctuations $[6,7]$.

The recent extension of the classical theory of fluctuations to nonequilibrium processes $[8,9]$ showed that the temporal relaxation of fluctuations could be directly obtained from fluctuation images. Such an approach led to experimental advances in measuring thermal diffusivity coefficient and viscosity coefficient. The recent generalization of DDM to investigating the dynamics of nonequilibrium fluctuations was introduced by Cerbino and Vailati $[1,2,3,4]$. DDM has also been applied to investigating equilibrium fluctuations close to critical con- 
ditions in binary mixtures [10] and under nonequilibrium conditions in dense colloids [11]. DDM method allows quantitative investigation of fluctuations in the fluid outside thermodynamic equilibrium, e.g., thermal, concentration, or density gradients. DDM also allowed low wavenumber range investigation where gravity dominates the dynamics of fluctuations, and new propagation modes influences by viscosity and gravity were observed [7, 12]. The information regarding the evolution and the scaling of the fluctuation relaxation time is contained in the Intermediate Scattering Function (ISF). For a pure fluid in thermal equilibrium, the ISF is a Gaussian with width proportional to the diffusion time. There are cases when ISF contains multiple time scales, and one approach has been the fitting of ISF with multiscale exponentials to capture so each characteristic time separately $[13,14]$. This approach allowed, for example, the separation of the thermal diffusivity coefficient from the mass diffusivity $[13]$.

However, when the experiments are performed within $\mu \mathrm{K}$ macroscopic finite time, and the finite size of the fluid observations can affect wavenumbers whose time and length characteristics are substantially different. The effect of multiple temporal and spatial scales that governed the energy transfer between probing photons and probed molecular systems is reflected in the existence of multiple decay exponentials in the Intermediate Scattering Function (ISF) [13]. Therefore, the traditional approach to the multiscale analysis of the images is 40 by fitting the ISF with exponential functions with multiple characteristic times $[13,14]$.

Although there is always a tradeoff between parsimony and the goodness of fit, here our goal was to both achieve low parsimony and good accurate description of experimental data by separating the dominant spatial scale related

45 to critical density fluctuations with the characteristic size of the order of the correlation length from any other significantly different spatial scales. To achieve this goal, we used the Bidimensional Empirical Mode Decomposition (BEMD) algorithm for the multiscale separation of the original image in multiple Intrinsic Mode Functions (IMFs) images. After obtaining the IMF images, we applied 
the DDM method to each IMF image set, as described in [5].

In this paper, after the selected recalling of the main experimental setup features in sections 2 and a brief description of optical features in section 3.1, we focus the IMFs results in section 4. The first IMF for the shortest spatial scale is related directly to the critical density fluctuations above $T_{C}$ (section 4.1). The second IMF with coarser structures can be linked (section 4.2) to the initial stage of cluster formation and phase separation process (below $T_{c}$ ). The relaxation time of fluctuations in the third-order IMF presents no noticeable structure. Section 5 focuses on the possible mechanisms that could explain the multiscale results. The concluding remarks in section 6 compare the results of this multiscale analysis and the existing theoretical predictions for critical fluctuations. The two subsections of the Appendix briefly recall the main characteristic features of the DDM technique (section 7.1) and the BEMD method (section 7.2), with related references.

\section{Experiments: setup aspects}

Direct imaging of large density fluctuations near the liquid-gas critical point of $\mathrm{SF}_{6}$ in microgravity environment was performed with ALICE 2 facility [15] on-board the MIR space station [16]. A cylindrical sample with an inner diameter of $12 \mathrm{~mm}$ and a thickness of $4.34 \mathrm{~mm}$ was filled with electronic quality $\mathrm{SF}_{6}$ corresponding to $99.98 \%$ purity (from Alphagaz-Air Liquide). The fluid is sandwiched between two sapphire windows with a $10 \mathrm{~mm}$ thickness each. The cell was housed inside a large sample cell unit made of electronic copper, the latter itself housed inside a removable thermostat device. The thermal controller resolution was $\pm 10 \mu \mathrm{K}$, and the stability was better than $40 \mu \mathrm{Kh}^{-1}$ [16]. The fluid inside the cell was initially filled at the critical density with the scalar order parameter $M=\left(\rho-\rho_{c}\right) / \rho=(0.00 \pm 0.02) \%$. For a complete description of the ALICE 2 facility and these microgravity experiments, see [15].

In this automated pioneering microgravity experiment, the $\mathrm{SF}_{6}$ sample was initially homogenized at $1 \mathrm{~K}$ above its critical temperature. The temperature 
was steeply and slowly decreased in a series of successive, fast temperature quenches with logarithmically decaying amplitudes. Each thermal quench was followed by long temperature relaxation periods with logarithmically increasing durations, until crossing the critical temperature. The final decreasing temperature timeline consisted of a series of $0.3 \mathrm{mK}$ thermal quenches, with two hours relaxation period, performed from a few milliKevins in the one-phase region above the critical temperature (see Fig. 1A). In addition to this automated temperature monitoring of the SCU, the fluid temperature was also measured with three small thermistors placed inside the fluid sample volume cell, which gave local fluid temperature status at two acquisition rates: $25 \mathrm{~Hz}$ and $2 \mathrm{kHz}$.

Hereafter we are only interested in the sample observation recorded during the last, fast $0.3 \mathrm{mK}$ thermal quench, which starts from the homogeneous domain above the critical temperature to finish into the two-phase region below critical temperature [5, 17], as shown in the inset of Fig. 1A. The full description of this last temperature quench was already presented elsewhere [5, 17]. The fluid volume observation during the two hours of the relaxation period following the final $0.3 \mathrm{mK}$ temperature quench revealed unambiguous nonhomogeneous liquid and gas domains within the sample fluid image, which were due to the $\mathrm{SF}_{6}$ phase separation. This two-phase state was evidenced by comparing against the homogeneous full image of the cell after the temperature relaxation period of the previous $0.3 \mathrm{mK}$ temperature quench. The critical temperature resulted somewhere between the upper (UP, i.e., $T>T_{c}$ ) plateau and the lower (DOWN, i.e., $T<T_{c}$ ) one (Fig. 1), and its exact location was previously determined with the histogram method in [17] and by using DDM in [5]. 

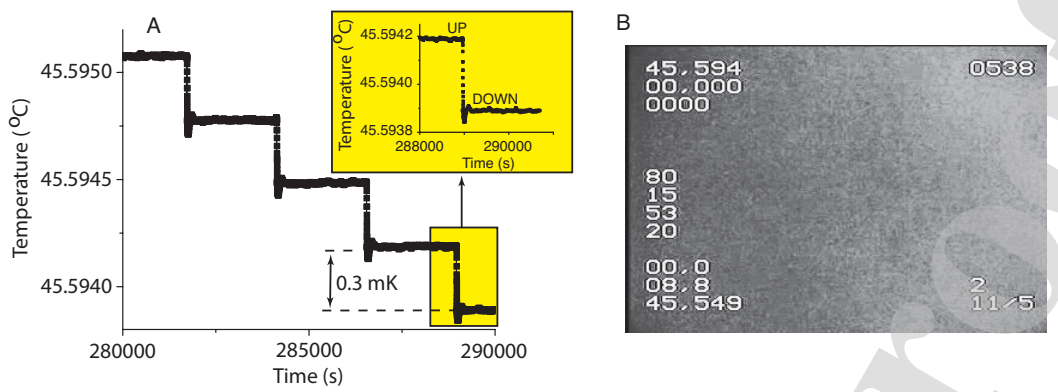

Figure 1: Multiple $0.3 \mathrm{mK}$ temperature quenches cooled down the supercritical fluid and eventually stepped through the critical point (see the inset). (A) The last $0.3 \mathrm{mK}$ temperature quench that stepped through $T_{c}$ has the temperature marked in Celsius degrees versus time in seconds (measured from the beginning of the experiment). (B) ALICE 2 marks each frame with a set of control numbers regarding the measured temperature, elapsed time, frame index, etc. For example, the left middle set of numbers gives the time elapsed since the beginning of the experiment: $80 \mathrm{~h} 15 \mathrm{~min} 53 \mathrm{~s}$ and $20 / 25$ (there are 25 frames per second).

\section{Experiments: Optical aspects and image processing}

\subsection{Optical aspects}

The following description of the optical characteristics refers to the 30 years old technologies used in the ALICE 2 facility here recalled anticipating a possible application of the BEMD method to the upgraded images which can be recorded from the real-time monitoring of the current and future similar experiments performed with the DECLIC and DECLIC-EVO instruments on-board of the International Space Station (ISS) [18, 19, 20, 21, 22, 23, 24, 25].

ALICE 2 has a modular optical design with a "source optical box" containing the laser, different filters, and photodiodes, the "thermostat box" that includes the sample cell unit, and a "collecting optical box" that contains the CCD and additional photodiodes [16]. The complete optical scheme is detailed in [15], and the optical performances are precisely analyzed for other experiments, such as turbidity measurements [16].

The fluid sample cell is visualized through light transmission normal to the windows using LED illumination with a spectrum centered around $660 \mathrm{~nm}$. A 
$632.8 \mathrm{~nm}$ He-Ne laser with about $1 \mathrm{~mW}$ maximum power is also used for other purposes (turbidity measurements). Laser stability after $1 \mathrm{~h}$ was estimated to be better than $0.3 \%$. ALICE 2 instrumentation works with a wide field of view $\left(10 \times 10 \mathrm{~mm}^{2}\right.$ object image $)$ at $30 \mu \mathrm{m}$ resolution and an additional microscope objective at $3.5 \mu \mathrm{m}$ resolution. All images analyzed in this study were obtained with the optical microscope, $3.5 \mu \mathrm{m}$ resolution, that visualized a small $0.9 \mathrm{~mm} \times 0.9 \mathrm{~mm}$ object image in the medium focal plane of the fluid layer, centered on the optical axis of the fluid sample and recorded at 25 frames per second. Each image contained the selected recording time marker, e.g., $80 \mathrm{~h}$ $15 \mathrm{~min} 53 \mathrm{~s}$ and frame 20 of 25 (288953.80 s) for the image illustrated in Fig. 1B corresponds to an image frame in the middle of the UP region. Instead of carrying over all the time digits, the original images used in this study were ordered in two disconnected sets where time origin corresponds to the first image of the first set. The UP image set contains 160 images recorded during the stationary temperature plateau above the critical temperature $\left(T>T_{c}\right)$. That corresponds to a period covering the range 0-6.4 s, before the beginning of the temperature quench (see Fig. 2). For the first 87 frames, the microscope focused on the sapphire windows (Fig. 2B). For the rest of the images, the focus plane was in the middle of the cell (Fig. 2C). The second set, named DOWN, contains 500 images recorded in the final transient period of the temperature quench, where the fluid temperature is just below the critical temperature $\left(T<T_{c}\right)$. That corresponds to a period covering the range $25.5 \mathrm{~s}$ to $44.6 \mathrm{~s}$, which consists of the very early stage of the expected phase separation process (see Fig. 2). For all DOWN images, the microscope focused in the middle of the cell.

Fig. 2 shows two original images $(\mathrm{B}, \mathrm{C})$, selected from the UP set $\left(T>T_{c}\right)$ and two original images $(\mathrm{D}, \mathrm{E})$ selected from the DOWN set $\left(T<T_{c}\right)$, with their corresponding position along the temperature timeline marked with B, C, D, and E in Fig. 2A. This figure illustrates the noticeable optical similarity of the grey level structures both for UP and DOWN regions. The first two images (panels B and C) reflect the contribution of the critical density fluctuations above $T_{c}$, where the fluid is in its homogeneous, one-phase equilibrium state. 


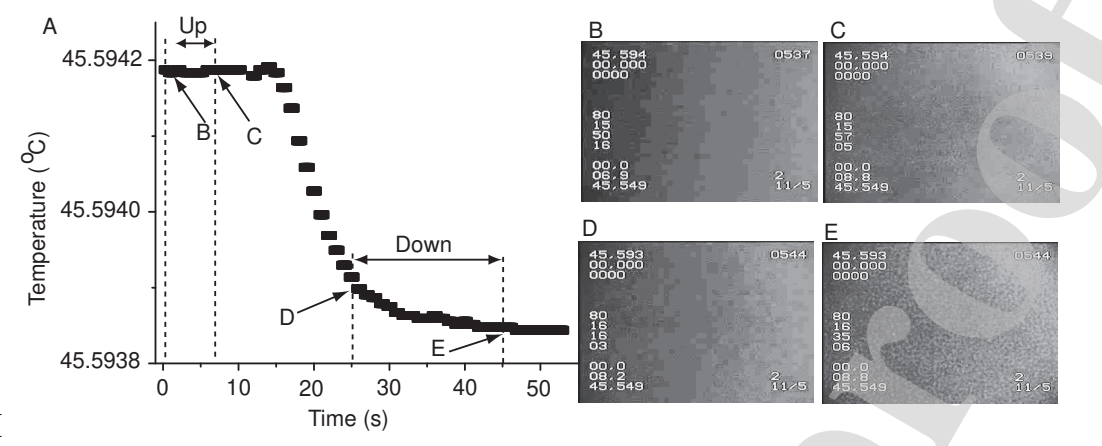

Figure 2: An enlarged version of the thermal quench of $0.3 \mathrm{mK}$ that steps through the critical temperature (A). The first image on the UP plateau (B) is the time reference (the horizontal axis in panel A). Fluctuation images taken on UP thermal plateau (B and C) show no phase separation. Fluctuating images at the beginning (D) and end (E) of the region called DOWN show that clusters start forming in this early stage of phase separation.

The other two images (panels D and E) are recorded during the early stage of the expected nucleation and growth of the two-phase domains below $T_{c}$ (see also [5]). The distinction is thus essential because the physics behind these two phenomena is very different: one is stationary, and the other is transient.

For the subsequent image analysis, the recording time marker shown in Fig. 1B, and all other tags, were eliminated by cropping the most extensive possible rectangular area of the image. Here, we have maximized the useable image size cropped from the original image of $352 \times 240$ pixels $^{2}$ by using $188 \times 238$ pixels $^{2}$ (total 44744 pixels) instead of the $192 \times 192$ pixels $^{2}$ (total 36864 pixels) square images cropped out the original image in [5]. As a result, the image analysis focused on a $0.66 \times 0.83 \mathrm{~mm}^{2}$ central region of the fluid sample.

For the Fourier spectra calculations, we should also distinguish between the number of pixels of the CCD sensor $N_{\text {pixel }}$ and the number $N$ of points of the Fast Fourier Transform (FFT). The $N$-point FFT has a frequency domain resolution of $\frac{2 \pi}{N \Delta x}$ and covers the wavenumbers uniformly from the shortest $q_{\text {min }}$, which corresponds to the largest spatial size of the image $l_{C C D}=N_{\text {pixel }} \times \Delta x$, to the largest possible wavenumber that corresponds to the smallest possible resolved distance in the image, i.e., $\Delta x$. The minimum possible wavenumber 
in a Fourier transform corresponds to the largest possible spatial dimension of the image, i.e., $q_{\min }=2 \pi / w$, where $w$ is the smallest side of the image. In our experiments, $w=$ magnification $\times l_{C C D}$, where magnification is the magnification factor of the microscope (in our case, 0.882), and $l_{C C D}$ represents the size of the image formed on the CCD sensor. With $l_{C C D}=N_{\text {pixel }} \times 3.5 \mu \mathrm{m}$ $=188$ pixels $\times 3.5 \mu \mathrm{m}=0.658 \mathrm{~mm}$, and magnification $=0.882$ one obtains $q_{\text {min }} \approx 108 \mathrm{~cm}^{-1}$. In the following, the dimensionless wavenumber is simply $175 \quad q *=q / q_{\min }$.

In this study, we increased the resolution of FFT from 256 points in [5] to 1024 points in this study. Since the linear size of images is over 128 but less than 256 pixels, we could use again (as in [5]) the 265 point FFT. Here, we used a 1024-point FFT to add more details to our construction of the structure factor and its relaxation time. The reason is that we wanted a better, more accurate, estimate of the thermal diffusivity coefficient above $T_{c}$, before applying the same approach in the case of an effective diffusion coefficient below $T_{c}$. The distinction is essential because very near, but above $T_{c}$, there is a single component system where thermal diffusion of the stationary density fluctuations is the dominant phenomenon. Very close, but below $T_{c}$, the system is the heterogeneous site of nonequilibrium relaxation processes for which an effective diffusion coefficient can be defined.

Due to its radial symmetry in the wavenumber space $\left(q_{x}, q_{y}\right)$, only the radial average of the power spectrum versus the magnitude of the wavenumber $190 q=\sqrt{x_{x}^{2}+q_{y}^{2}}$ was considered. The results obtained for small wavenumbers are affected by significant errors because of the small azimuthal radius, which determines a poor ensemble statistical average. Due to the larger cropped area and the increased FFT resolution, the number of statistically independent samples at the maximum wavenumber increased from 400 in Ref. [5] to over 2000 in this study. Indeed, at large wavenumbers, the number of pixels that act as independent samples in a single ring of the azimuthal average of the power spectrum increases as $\pi n$, in which $n$ is the number of the channel varying from 1 to $N / \sqrt{2}$. 


\subsection{Image processing using Dynamic Differential Microscopy (DDM) technique}

This section reviews the main points about the DDM method, especially its application in the image processing above $T_{c}$, as used in our previous work [5]. From the Fourier spectra of successive differences between images taken at a fixed delay time $\delta t$, it was possible to determine the structure factor of critical density fluctuations and their relaxation time $[7,12,26]$, which allowed us to determine the thermal diffusivity coefficient [5, 17]. Theoretically, the structure factor $S(q)$ of the critical density fluctuations in a homogeneous singlecomponent fluid near its critical temperature is expected to be a Lorentzian:

$$
S(q)=\frac{1}{\left(1+q^{2} \xi_{+}^{2}\right)},
$$

where $\xi_{+}$is the correlation length of the fluctuations above the critical temperature $\left(T>T_{c}\right)$, and $q=2 \pi / l$ is the wavenumber with $l$ being the linear dimension of the fluctuating domains [27]. The typical relaxation time $\tau$ of critical fluctuations above $T_{c}$ should obey Kawasaki [28] formula:

$$
\tau^{-1} \approx D q^{2}
$$

when $\xi_{+} q<<1$ (hydrodynamic regime), where $D$ is the thermal diffusivity coefficient. On the other hand, for $\xi_{+} q>>1$ (critical regime), this relaxation time should scale as:

$$
\tau^{-1} \approx A q^{3}
$$

with $A=$ constant $[28,29,30]$.

To confirm that the experiments were performed in the hydrodynamic regime, we estimated the correlation length for each experimental temperature. The correlation length is given by $\xi=\xi_{+}\left(\left(T-T_{c}\right) / T_{c}\right)^{-\nu}$, with $\xi_{+}=1.8 \times 10^{-10} \mathrm{~m}, T_{c}$ $=318.733 \mathrm{~K}$, and $\nu=0.6304[16,31]$. For the stationary temperature plateau above $T_{c}$ before the $0.3 \mathrm{mK}$ temperature quench, the temperature distance to $T_{c}$ was previously estimated to be $270 \mu \mathrm{K}[5,17]$. The correlation length is then $\xi \approx 10^{-4} \mathrm{~cm}$. 
The following step needs to recall our previous estimation [5, 17] of the characteristic size $l$ of the observed fluctuating density domains, precisely based on the relaxation time obtained from the DDM method and shown in Fig. 3. This experimental result was similar to part (b) of the theoretical Fig. 3 of Ref. [5], which is described by the above theoretical Eq. 1. We also must notice that the measured decreasing rate of the relaxation time at large values of $q$ was slightly greater than the theoretical slope of -2 suggested by Eq. 1, as shown by the dashed line of slope -2.3 in Fig.4b of Ref. [5]. The location of the relaxation time peak defines the critical wavenumber $q_{c}$. In this experiment, $\xi_{+} q<<1[5,17]$, which means that the fluid was in the hydrodynamic regime. Therefore, the relaxation time of the density fluctuations should obey Eq. 1 for wavenumbers $q>q_{c}[28]$.

To capture the behavior of the relaxation time over a broader range of wavenumbers, on both sides of the critical wavenumber $q_{c}$, we fitted the relaxation time of fluctuations with $[6,7]$ :

$$
\tau^{-1}=D q^{2}\left(1+\left(\frac{q_{c}}{q}\right)^{4}\right),
$$

which reduces to Eq. 1 for large wavenumbers. When using Eq. 2, one can extract both the diffusion coefficient $D$ and the critical wavenumber $q_{c}$. Such results are exemplified in Fig. 3, where the continuous green curve corresponds to fitting the solid black square (image set B from Fig. 2), and the solid blue line corresponds to fitting the solid red circles (image set $\mathrm{C}$ from Fig. 2). As noted in Fig. 3, $q_{c}$ remains constant, within the limits of the experimental errors, which means that the characteristic size of fluctuations is constant during the UP plateau. The caveat of fitting the data with Eq. 2 over the entire range of wavenumbers is that at low wavenumbers, the values of $\tau(q)$ are scattered due to poor data statistics (see Fig. 3). One could avoid such issues by only fitting the data at large wavenumbers with the reduced formula given by Eq. 1. In this case, the straight line with a slope of -2 (see the continuous white line in Fig. 3) gives the diffusion coefficient $D$. In addition to the above direct fitting of the relaxation time data to Eq. 2, or the reduced Eq. 1, a quick estimate of 
the thermal diffusivity coefficient can also be obtained from the peak value of the relaxation time shown in Fig. 3. Indeed, at the peak of the relaxation time curve, $\left(\tau=\tau_{\max }, q=q_{c}\right)$ and Eq. 2 reduces to:

$$
\tau_{\max }=\frac{1}{D q_{c}^{2}}
$$

$255 \quad$ with $D=\frac{1}{\tau_{\max } q_{c}^{2}}$ as a resulting value.

As shown in [5], it was also possible to repeat a similar fitting procedure of the relaxation time obtained from the DDM method applied to the DOWN set of original images recorded below $T_{c}$, where the approximated correlation length is $\xi_{-}=\xi_{+} / 2$. However, it was essential to note that the resulting fitted values of $D$ correspond then to the ones of an effective diffusion coefficient related to the fluid physical behaviors that are undoubtedly nonstationary. 


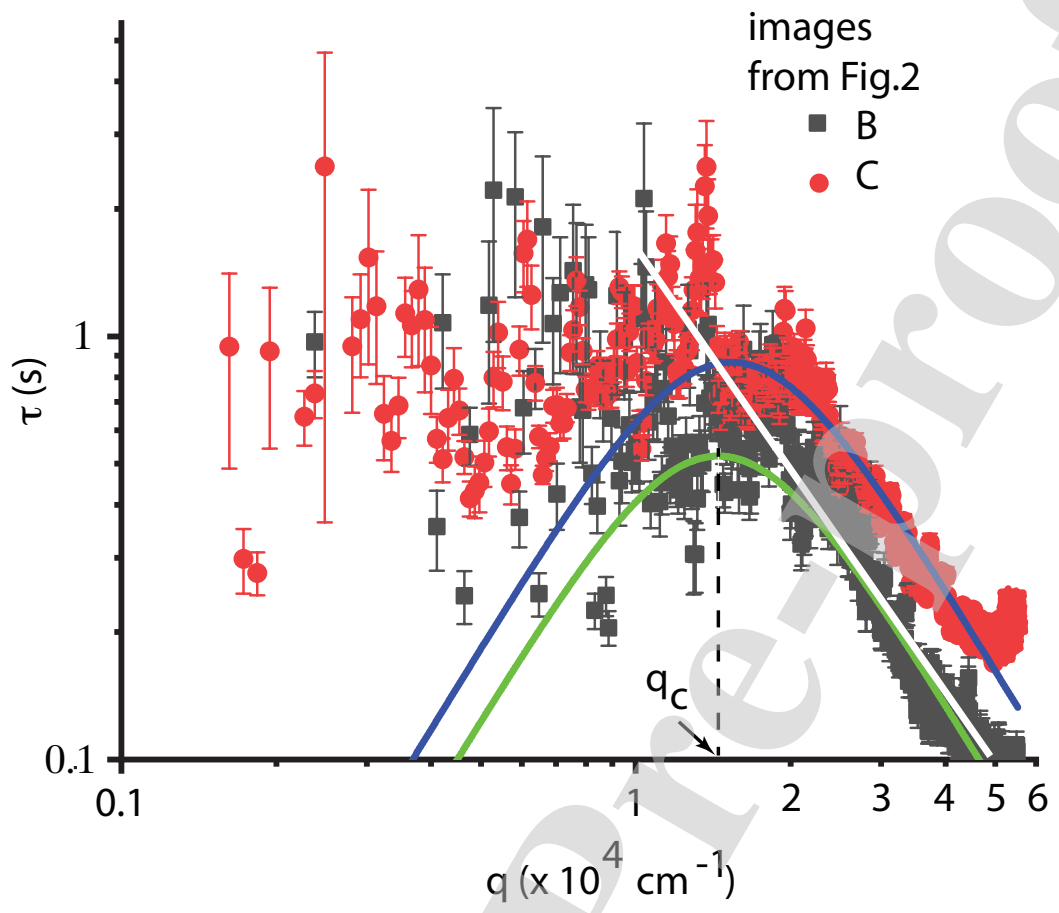

Figure 3: Correlation time of fluctuations for the two representative images from the UP region, i.e., images B (solid black squares) and C (solid red circles) from Fig. 2. The peak of the correlation time $\tau$ determines the critical wavenumber $q_{c}$. At low wavenumbers, the data are significantly scattered due to poor statistics. The fitting curve from Eq. 2 is shown with a continuous green line overlapped with the image set B (solid black squares). For wavenumbers larger than $q_{c}$, the log-log plot can be approximated with a straight line (see the white line overlapped with the image set B) with a slope of -2 as predicted by Eq. 1 .

\section{Results}

4.1. Main features of the image processing using the DDM method applied to the UP set

The above applications of the DDM method were mainly based on the final determination of the relaxation time versus the scattered light's wavenumber. We note, however, that the experimental decay of the relaxation time is not precisely matching the expected theoretical one, as shown in Eq. 2. 
Our experimental determination of time-dependent structure functions $C_{m}(q, \delta t)$ $S T(q)$ does not match the expected theoretical function over the entire range. For example, at low wavenumbers, the experimental structure factor starts diverging, while the theoretical prediction shows an almost constant $S T(q)$. As a direct consequence, it seems possible to break this theoretical modeling introclearly shows that it saturates for a delay time $\delta t$ below one second (see Fig. $4 \mathrm{~A}$ ) The time-dependent structure functions $C_{m}(q, \delta t)$ shown in Fig. 4A described how the spectral power changes with the delay time $\delta t$ between images for a fixed wavenumber $q$, and it is given by:

$$
C m(q, \delta t)=2(S(q) * T(q) *(1-G(q, \delta t))+B(q)),
$$

where $S(q)$ is the structure factor of the fluctuations, $T(q)$ is the transfer function of the optical setup, $B(q)$ is a background contribution to structure factor, and $G(q, \delta t)$ is the Intermediate Scattering Function (ISF). Unless the optical transfer function $T(q)$ is explicitly given, the DDM method can only determine the product $T(q) * S(q)$, which we called $S T(q)$. The corresponding Intermediate Scattering Function (ISF) of the image set C, shown in Fig. 4A has an apparent linear decay (in linear-log axis) for the small delay time (below one second) and a slightly different decay exponent afterward (see Fig. 4B). The experimental structure factor $S T(q)$ from the UP set of original images (Fig. 4C) appears similar to the theoretical prediction of Fig. 3a in Ref. [5]. Similarly, the experimental relaxation time of fluctuations $\tau$ shown in Fig. 4D follows the theoretical prediction from [5]. As predicted theoretically, at large wavenumbers $(200 \leq q * \leq 400)$, the relaxation time follows a power law, which is suggested in the log-log plot shown in Fig. 4D by the straight lines with slopes of $-2.15 \pm$ 0.04 (continuous white line in Fig. 4D) and $-1.91 \pm 0.04$ (black dashed line in Fig. 4D) with adjusted $R^{2}=0.999$ for each fitting. Based on the above theoretical description [5], it is also noticeable that the wavenumber dependence of ducing (at least) one more different temporal or spatial scale exponents. 

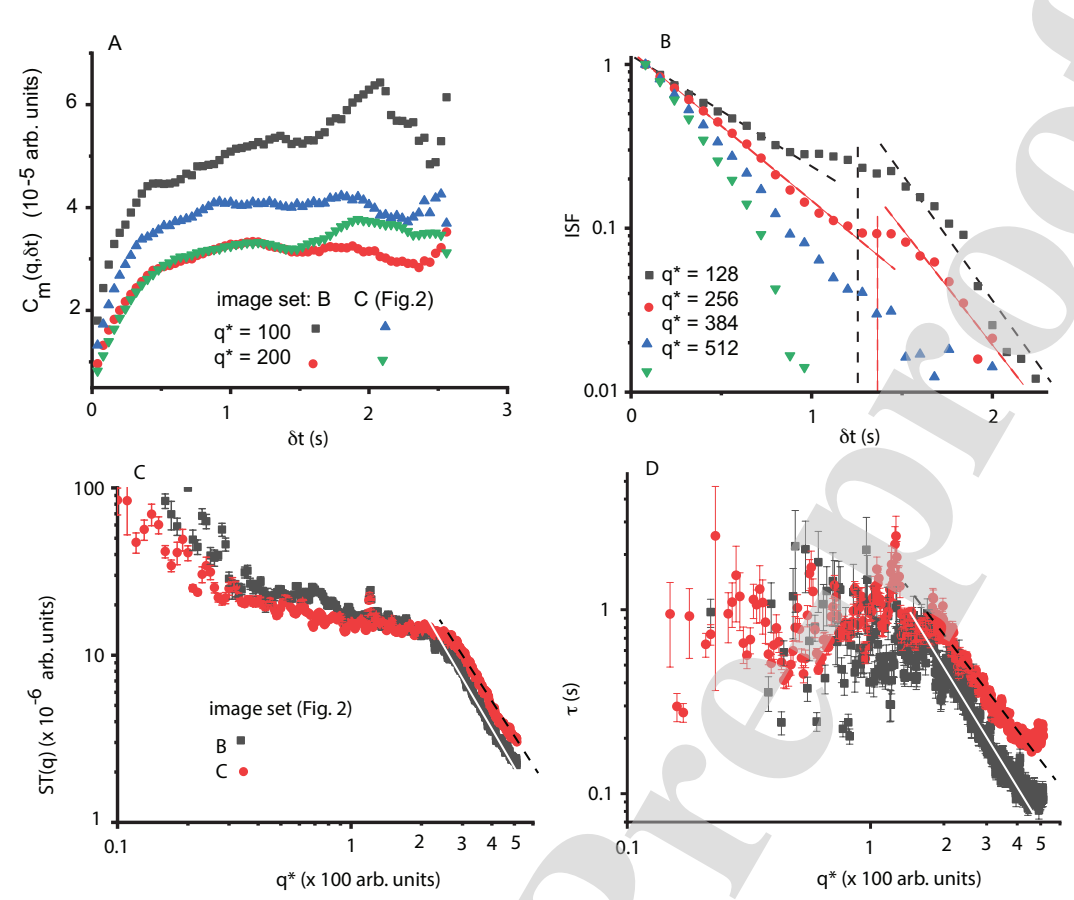

Figure 4: The time-dependent structure functions $C_{m}(q, \delta t)$ saturates after a relatively short delay time $\delta t$ between successive images (A). The saturation delay time is determined by the relaxation time of fluctuations and depends on the wavenumber. The two representative examples of the UP region correspond to images B and C shown in Fig. 2. (B) The linear-log plot of the Intermediate Scattering Function (ISF) $G(q, \delta t)$ of the image set C from panel A shows that it may be possible to capture its shape with two decaying exponentials. The dashed lines suggest the best fits with exponential functions across different regions of the delay time $\delta t$. The inflection point, marked by the vertical dashed lines, depends on the wavenumber. (C) The log-log plot of the structure factor $S T(q)$ shows a power-law dependence with a slope of approximately -2 for large wavenumbers, i.e., $q *>250$, which corresponds to $q=q * \times q_{\text {min }}=$ $250 \mathrm{~cm}^{-1} \times 108 \mathrm{~cm}^{-1}=27000 \mathrm{~cm}^{-1}$. At intermediate wavenumbers, $S T(q)$ has no structure, whereas it diverges at low wavenumbers due to poor statistics. (D) The relaxation time of fluctuations $\tau$ shows a clear power-law dependence at large wavenumbers $(200 \leq q * \leq 400)$ with the slopes close to the theoretically predicted value of -2 .

As we showed in [5], the theoretical structure factor is given by

$$
S(q)=\frac{S_{0}}{1+\left(\frac{q}{q_{c}}\right)^{2}},
$$


where $S_{0}$ is the structure factor value at very low $(q \rightarrow 0)$ wavenumbers, and $q_{c}$ is the critical wavenumber. In the $\log -\log$ plot, the theoretical $S(q)$ looks almost constant at low and intermediate wavenumbers and decays as a power-law with a -2 exponent for large wavenumbers (see Fig.3a in [5]). The experimental data in Fig. 4C is similar to theoretical predictions form [5], i.e., at large wavenumbers, the power-law exponent is $-2.45 \pm 0.02$ for image set $B$ (continuous white line overlapped with solid black squares in Fig. 4C) and $-2.34 \pm 0.02$ for image set $\mathrm{C}$ (black dashed line overlapping with the solid red circles). At intermediate wavenumbers, the structure factor $S T(q)$ is almost constant as predicted theoretically, whereas, at very low wavenumbers, the experimental structure factor diverges due to poor data statistics.

The saturation of time-dependent structure functions $C_{m}(q, \delta t)$ shown in Fig. $4 \mathrm{~A}$ and the dependence of the structure factor on the wavenumber $q$ provide additional insight into the characteristic relaxation time of the critical density fluctuations in the homogeneous domain (also compare with Fig. 3 of [5]). In such a theoretical framework of the critical phenomena, only a single characteristic time can govern the exponentially critical decaying of the ISF $G(\vec{q}, \delta t)$ from Eq. 3, as follows:

$$
G(\vec{q}, \delta t)=\exp \left(\frac{-\delta t}{\tau(q)}\right)
$$

When plotted in linear-log coordinates, the slope of Eq. 5 equals the inverse of the characteristic relaxation time of critical density fluctuations in the homogeneous domain (see Fig.4C). The experimental data show that the linear-log plots of the ISFs appear to have two different linear regions, which suggest that there may be two different relaxation times involved in the critical density fluctuations. To elucidate this aspect, we used the BEMD decomposition to understand the shape of the ISF better. 
4.2. Comparison with the main features of image processing using the BEMD algorithm applied to the UP set

The above observations reported in Figs. 3 and 4 suggest that fluid physics

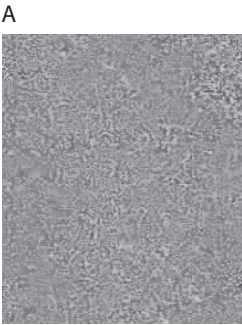

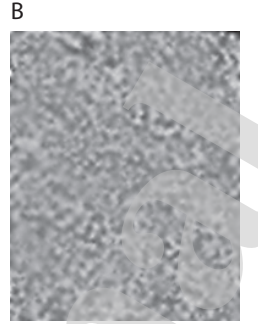

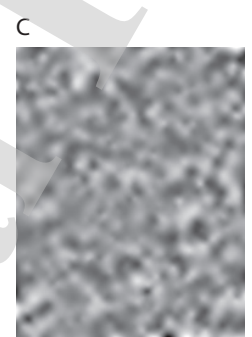

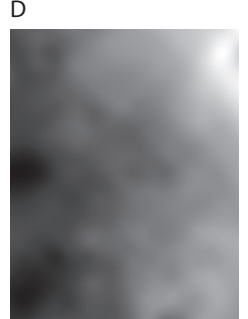

Figure 5: The first three IMFs for the $100^{\text {th }}$ image of the UP region are IMF1 (A), IMF2 (B), and IMF3 (C) with the residual image shown in panel D. Examples of original images are in Fig. 2B and C. Above the critical temperature, the finest spatial scale IMF1 (A) shows relatively small size fluctuations that correspond to the finest spatial scale of the fluctuations. The coarse spatial scales (B and C) show long-range correlation patterns of fluctuations. Usually, the residue (D) shows the amount of nonuniform illumination in the original image.

Therefore, we expect that the first mode associated with Fig. 5A corresponds to the dominant critical correlation length scale of the critical density fluctuations very close to the critical point. Conversely, any other unknown modes 
can be contained in the second IMF2 (Fig. 5b) and the third IMF3 (Fig. 5c). The residue shows the effect of the remaining nonuniform illumination of the original image (Fig. 5d).

We have shown in subsection 4.1 that the time scale separation suggested by the shape of the ISF of Fig. 4 results naturally from the data-driven multiscale separation of the original images produced by the BEMD algorithm. Therefore, applying the DDM method to each IMF, a corresponding ISF is obtained, which can then be described by a single exponential, as shown in Fig. 6 from the ISF versus the lag time $\delta t$ in linear-log diagrams. In Fig. 6, the first IMF contains similar information regarding the single characteristic time $(\tau)$ of the critical density fluctuations. Each ISF of Fig. 6 was fitted to a single exponential decay, and the resulting decay times are shown in Fig. 7 in a $\tau$ versus $q \log$-log diagram together with the relaxation time of fluctuations in the original image before BEMD decomposition (solid squares). The four panels in Fig. 6 correspond to the normalized wavenumbers shown in Fig. 4 C, i.e. $q *=128, q *=256, q *=$ 384 , and $q *=512$. While most ISFs can be fitted with a single exponent, for some wavenumbers, the original ISF seems to be better captured by two exponents, such as in Fig. 6A and B. We also notice in Fig. 6 that the exponents (slopes of log-linear straight lines) for the original images (slid squares) and IMF1 (solid circles) are significantly closer to each other than to higher-order IMFs. The slope of IMF3 (solid inverted triangles) and additionally, the spread of values for IMF3 (see Fig. 6) is significantly different from the reset of the ISFs, which lead us to ignore it at this step of the analysis. 

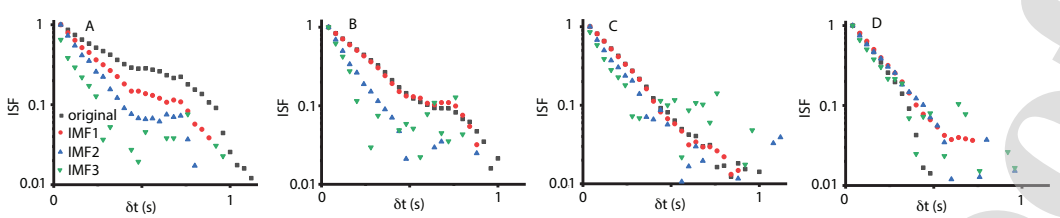

Figure 6: For each ISF of the original images shown in Fig. 4C, the corresponding ISF of the BEMD decomposition are given for different wavenumbers in $q *=128$ (A), $q *=256$ (B), $q *=384(\mathrm{C})$, and $q *=512(\mathrm{D})$. The linear-log plot of the Intermediate Scattering Function (ISF) versus the delay time $\delta t$ shows that it could be fitted with two different exponents for the original images, i.e., one for low delay times $\delta t$ and a larger exponent for longer delays. At large $\delta t$, the ISFs become scattered due to image de-correlation. This effect is exacerbated as the wavenumber increases, i.e., as the spatial scale of fluctuations decreases, suggesting that small fluctuations de-correlate over a smaller delay window.

In Fig. 7, we notice that the relaxation time of fluctuations in the original images (solid squares in Fig. 7) overlaps quite well with the relaxation time of fluctuations in the first IMF (solid circles in Fig. 7), at least for large wavenumbers (over $6000 \mathrm{~cm}^{-1}$ ). Their apparent decay slopes at large wavenumbers are also similar and slightly larger than -2 . Noticeable differences between the original data and IMF1 occur at low wavenumbers where the IMF1 is below the values of the original data's relaxation time but without distinction in the peak value and its related $q_{c}$ value. IMF2 (solid upside triangles in Fig. 7), as IMF3 (solid upside triangles in Fig. 7), reveal any characteristic dynamics associated with noticeable larger spatial structures, except that IMF2 presents a possible "bump" at low wavenumbers (below $5000 \mathrm{~cm}^{-1}$ ). We notice from Fig. 7 of the UP region that the correlation time of fluctuations in IMF3 is about $(0.09 \pm 0.02) \mathrm{s}$, representing two video frames. This means that the large-scale structures seen in Fig. 5C are short-leaved, and they dissipate quickly. The structures corresponding to IMF2 (see Fig. 5B) have a longer life span of $(0.15 \pm 0.03) \mathrm{s}$, representing four video frames. Finally, the shot-scale structures seen in IMF1 (Fig. 5A) have the longest life span of $(0.23 \pm 0.07) \mathrm{s}$, representing six video frames on average. These values represent the "background" correlation time of fluctuations for the respective IMFs. Close to the critical wavenumber $q_{c} \approx 8000 \mathrm{~cm}^{-1}$, 
the correlation time of fluctuations in IMF1 is longer than 10 frames. For such large structures, such as those associated with IMF2 and IMF3 (Fig. 5 B and C), to dissipate so quickly, they must have a relatively larger diffusion coefficient than small spatial scale and long-leaved structures from IMF1 (Fig. 5A). As a result, we can only observe the correlation time for the most persistent fluctuations when the DDM is applied to the original images that contain all three IMFs (plus the residual). The same is true for the DOWN region where the "background" correlation times $(0.14 \pm 0.03) \mathrm{s}$ for IMF2, and $(0.22 \pm 0.07) \mathrm{s}$ for IMF1, while the correlation time estimate for IMF3 was not reliable (see Fig. 10).

Furthermore, we also notice from Fig. 7 that the correlation time of fluctuations in the original images is the sum of correlation times extracted from individual IMFs:

$$
\tau_{\text {original }}=\tau_{I M F 1}+\tau_{I M F 2}+\tau_{I M F 3},
$$

and we conjecture that the relationship is valid for an arbitrary number of IMFs. 


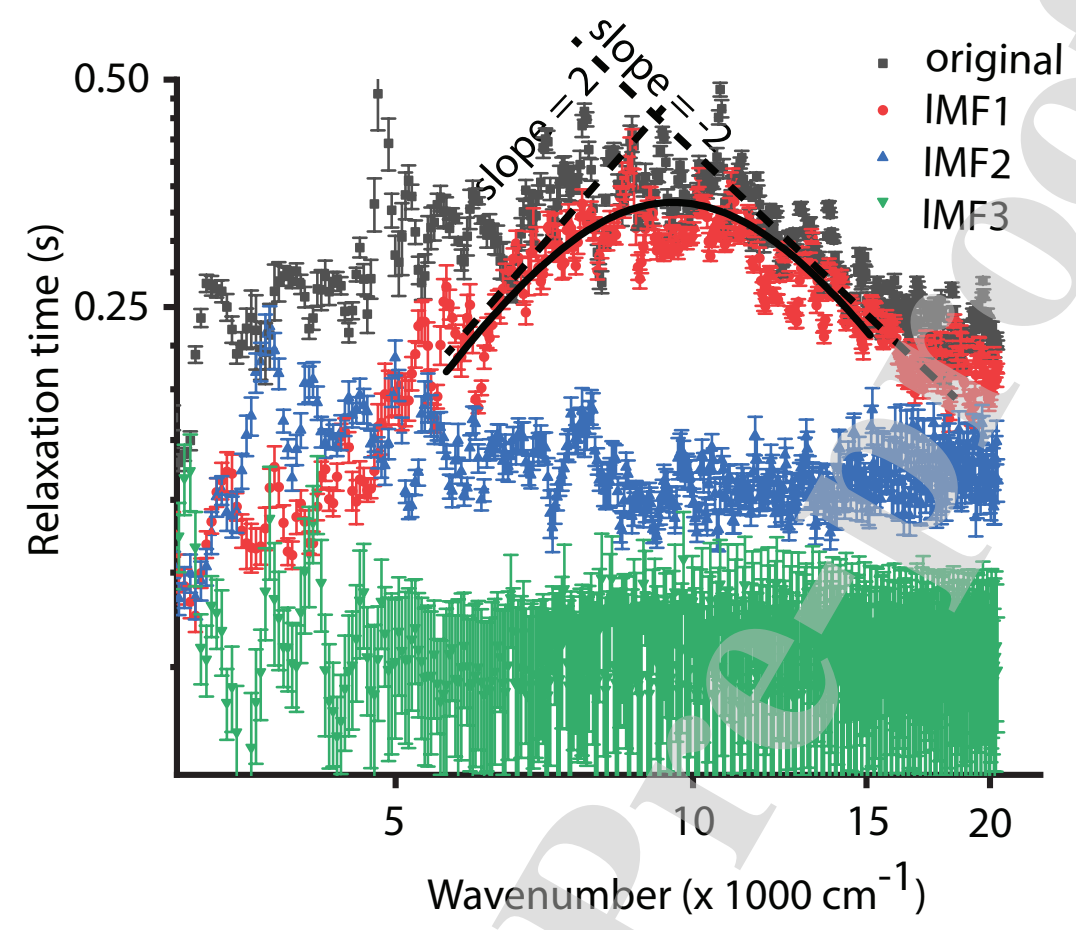

Figure 7: The relaxation time of fluctuations versus wavenumber for UP images starting around the snapshots shown in Fig. 2. The first IMF (solid circles) captures more than $90 \%$ of the shape of the relaxation time of fluctuations in the original image (solid squares). The second IMF (solid upright triangles) has a hard-to-distinguish "bump" at low wavenumbers. The third IMF has no structure (solid inverted triangles). As a result, the fluctuations can be described by only two time scales: one for very fine fluctuations in IMF1 and a coarser fluctuation in IMF2. The solid arches show the fitting curve given by Eq. 2 for the IMF1 and IMF2 around the peak wavenumber $q_{c}$.

\subsection{Extension of the BEMD method to the DOWN set}

In a similar use of the DDM method for the DOWN set of the original image, we have also extended the application of the BEMD algorithm to compute the three IMFs for this DOWN set. The results are presented in Figs. 8, 9, and 10 and are similar to the above Figs. 5, 6, and 7 for the UP set. 

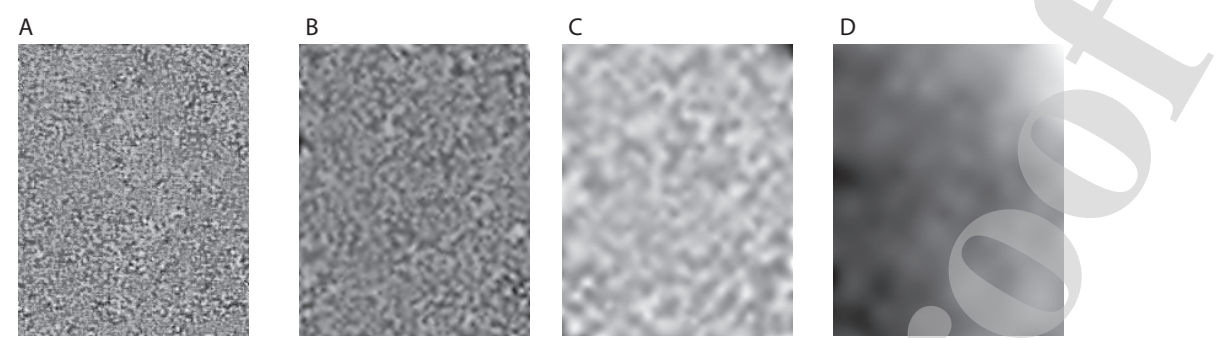

Figure 8: The first three IMFs for the $400^{t h}$ image of the DOWN region are IMF1 (A), IMF2 (B), and IMF3 (C) with the residual image shown in panel D. Examples of original images are in Fig. 2B and C. Above critical temperature (panels A-D), the finest spatial scale IMF1 (A) shows relatively small size fluctuations, which correspond to the finest spatial scale of the fluctuations. The coarse spatial scales (B and C) show long-range correlation patterns of fluctuations. Usually, the residue (D) shows the amount of nonuniform illumination in the original image.

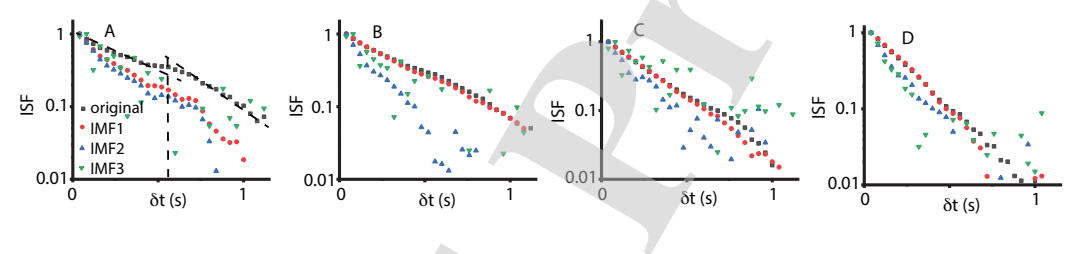

Figure 9: The lin-log plot of the Intermediate Scattering Function (ISF) versus the delay time $\delta t$ for DOWN region shows that it could be possible to fit the ISF of the original images (solid square) with two different exponents, i.e., one for low delay times $\delta t$ and a larger exponent for large delays. At large $\delta t$, the ISFs become scattered due to image de-correlation, although the transition is smoother than for the UP region shown in Fig. 6. Additionally, the de-correlation tends to occur around the same delay $\delta t$ across different wavenumbers. For each ISF of the original images shown with solid squares, the corresponding ISF of the BEMD decomposition are given for different wavenumbers in $q *=128(\mathrm{~A}), q *=256(\mathrm{~B}), q *=384(\mathrm{C})$, and $q *=512$ (D). 


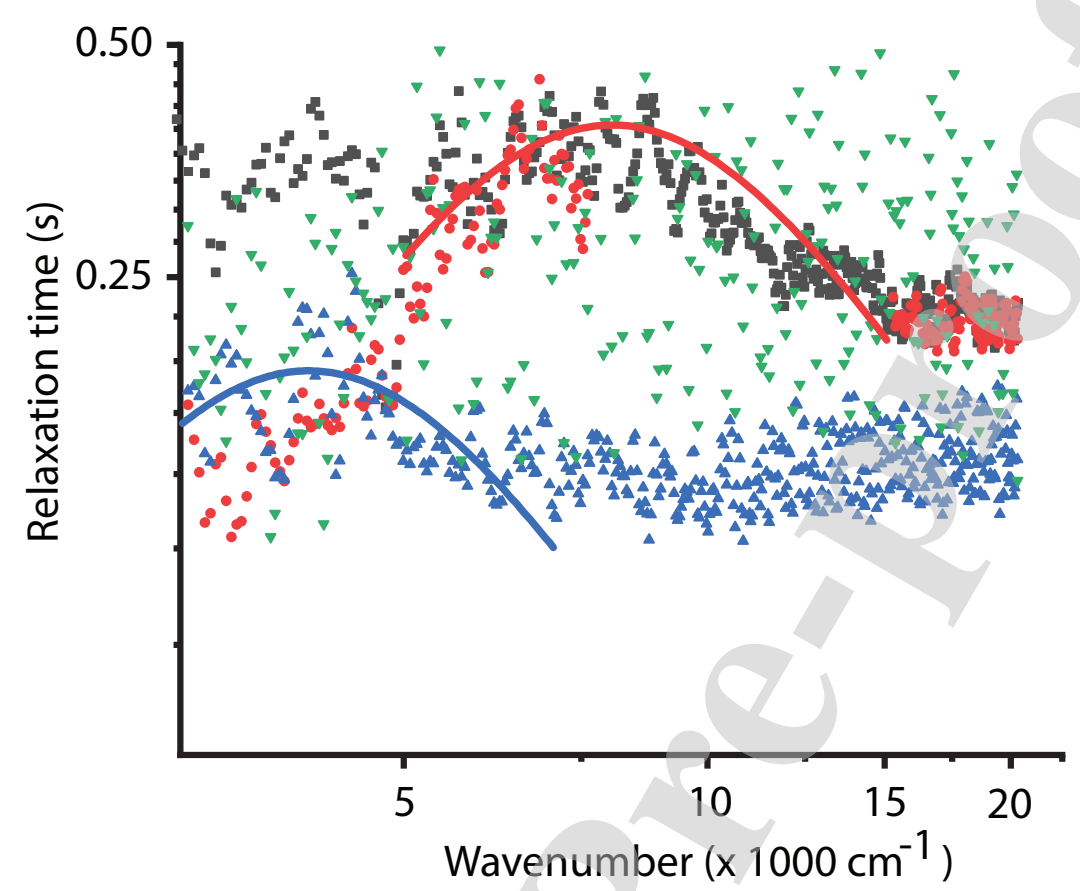

Figure 10: The relaxation time of fluctuations versus wavenumber for DOWN images starting around the snapshots shown in Fig. 2. The first IMF (solid circles) captures more than $90 \%$ of the shape of the relaxation time of fluctuations in the original image (solid squares). The second IMF (solid upright triangles) has a hard-to-distinguish "bump" at low wavenumbers. The third IMF has no structure (solid inverted triangles), which suggests that two time scales can describe the fluctuations: one for very fine fluctuations in IMF1 and a coarser fluctuation in IMF2. The solid arches show the fitting curve given by Eq. 2 for the IMF1 and IMF2 around the peak wavenumber $q_{c}$. The error bars are only shown in Fig. 7 for data of the UP region.

We need to make two notes regarding the data fitting shown in Fig. 10: First, we found that $q_{c}$ slowly drifts towards smaller values [17], suggesting that the characteristic length $l$ of the fluctuating clusters increases and leads to phase separation. Below $T_{c}$, the critical wavenumber $q_{c}$ corresponds to the size of phase separating domains. Our previous analysis showed that $q_{c}$ slowly decreases over time almost linearly and that $\xi_{+} q<<1[5,17]$.

Second, as we notice from Fig. 10, the peak of IMF2 shifted to about 
$3000 \mathrm{~cm}^{-1}$, a lower wavenumber than to the $8000 \mathrm{~cm}^{-1}$ in IMF1. However, because the wavenumber contribution to diffusivity/diffusion coefficient is squared, the above 2.7 times wavenumber decrease from IMF1 to IMF2 translates into a seven-fold increase of D. Additionally, there is a decrease in the relaxation time between IMF1 and IMF2 by a factor of 2 . As a result, the combined effect of simultaneous decreased peak relaxation time $\tau_{\max }$ and peak wavenumber $q_{c}$ is an increase by about an order of magnitude of the diffusivity/diffusion coefficient obtained from IMF2 compared to IMF1 (see Fig. 11).

The "bump" at low wavenumbers for IMF2 is very hard to separate from the background noise. Indeed, for IMF1 (see, for example, Fig. 10A), the background relaxation time at very large wavenumbers is about $0.207 \pm 0.016 \mathrm{~s}$ while the "bump" of IMF1 is at around 0.372 s. Even by subtracting three standard deviations, the peak relaxation time decreases from $0.372 \mathrm{~s}$ to $0.324 \mathrm{~s}$, which is still well above the background value of $0.207 \mathrm{~s}$. In other words, the "bump" of IMF1 is well-defined and statistically significant. However, for IMF2, the background (almost constant) value of the relaxation time at very large wavenumbers is about $0.15 \mathrm{~s} \pm 0.01 \mathrm{~s}$ while the "bump" of IMF2 is at around $0.18 \mathrm{~s}$. In this case, subtracting three standard deviations from the $0.18 \mathrm{~s}$ peak value of the relaxation time leaves us at the background noise level. While three standard deviations above the background is a reasonable ground for fitting the relaxation time of IMF2 data with Eq. 2, the errors on the diffusivity/diffusion coefficients are systematically higher than for IMF1 (see Fig. 11). 

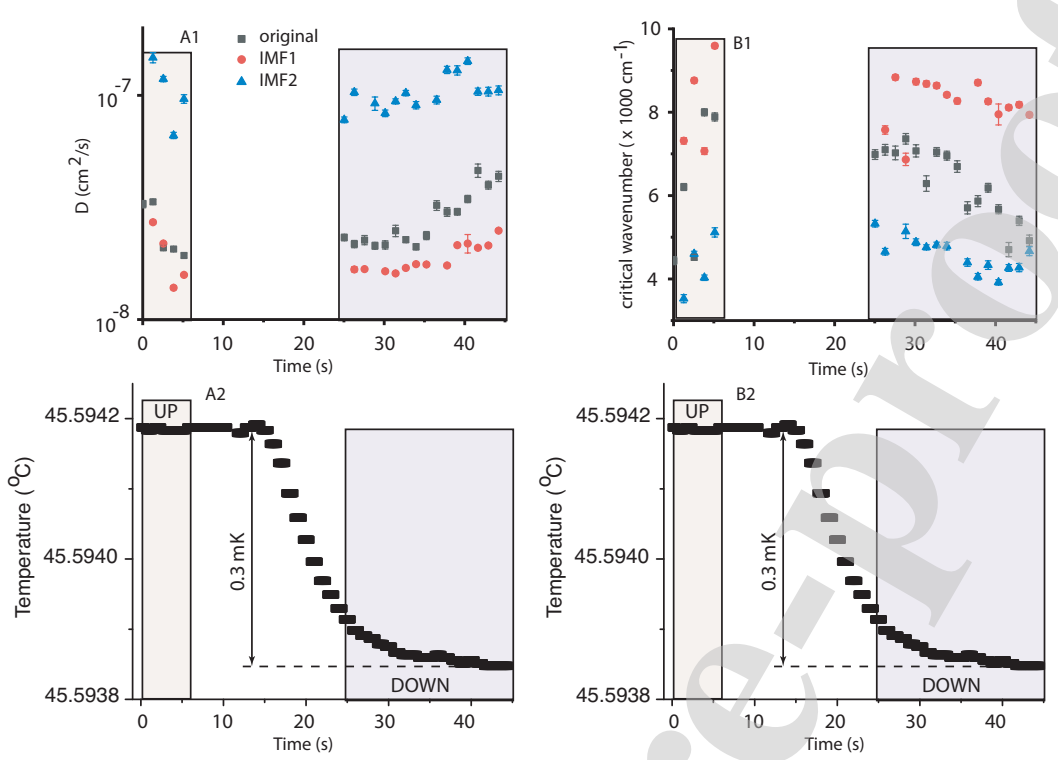

Figure 11: Diffusivity (above $T_{c}$ ) and effective diffusion/coefficient (below $T_{c}$ ) (A1) and the critical wavenumber (B1) for the original images (solid square), IMF1 (solid circles), and IMF2 (solid triangles). The diffusivity/effective diffusion coefficient obtained from the original image is consistent with previous results [5]. The diffusivity/effective diffusion coefficient for the finest fluctuations (solid circles in A) in the images is close to the values from the original images (solid squares in A) as they dominate the density fluctuations. There is almost one order of magnitude larger diffusivity/effective diffusion coefficient (solid triangles) associated with the long-range correlated structures. The finest fluctuations in the system have the largest critical wavenumber (solid circles in B), consistent with the fact that the wavenumber is inversely proportional to the length scale of fluctuations. The origin of time is the crossing of $T_{c}$, i.e., the negative time values refer to UP plateau $\left(T>T_{c}\right)$, and positive time values indicate DOWN plateau $\left(T<T_{c}\right)$. The lower panels show the ideal temperature profile. The UP and DOWN rectangles on the temperature profile corresponding to rectangles shown in panels A1 and B1 (the actual temperature profile measured by thermistors inside the fluid is shown in Fig. 3A.

\section{Discussion}

Should there be any separate spatial scale IMF2 above $T_{c}$ ? We investigated the possibility that the values shown in Fig. 11A as diffusion coefficients for IMF2 (see the solid triangles) could be related to a viscosity mode. Previously 
studies on nonequilibrium systems under concentration and gravity gradients $[7,12]$ used a more general relaxation time of fluctuations than Eq. 2:

$$
\tau=\frac{1}{D q^{2}}\left(1+\left(\frac{q_{c}}{q}\right)^{4}\right)^{-1}+\frac{2}{\eta_{\text {kinematic }} q^{2}},
$$

where $\eta_{\text {kinematic }}$ is the kinematic viscosity. Based on [32], we estimated the value of the dynamic viscosity near the critical point (see Eq. 6.8 in [32]):

$$
\eta_{\text {dynamic }}=\eta_{0}\left(Q_{0} \xi_{0}\right)^{y / \nu} \epsilon^{-y}
$$

where $\eta_{0}=(39.9 \pm 0.4) \mathrm{\mu Pas}$, with $\left(Q_{0} \xi_{0}\right)^{-y / \nu}=1.30 \pm 0.04$ for $\mathrm{SF}_{6}$, and $y=$ 0.04. For a temperature quench of $T-T c=0.3 \mathrm{mK}$ and a critical temperature of $T_{c}=(318.769 \pm 0.003) \mathrm{K}$, the reduced temperature $\epsilon=(T-T c) / T c=9.411 e-7$ gives $\eta_{\text {dynamic }}=5.347 \times 10^{-5} \mathrm{Pas}$. The corresponding kinematic viscosity is $\eta_{\text {kinematic }}=\eta_{\text {dynamic }} / \rho_{c}=7.324 \times 10^{-8} \mathrm{~m}^{2} / \mathrm{s}$ with $\rho_{c}=(730 \pm 7) \mathrm{kg} / \mathrm{m}^{3}$. Even if the critical temperature distance $T-T c$ is between $46 \mu \mathrm{Kand} 270 \mu \mathrm{K}$ as predicted in $[17,5]$, the kinematic viscosity would be in the range $7.895 \times 10^{-8} \mathrm{~m}^{2} / \mathrm{s}$ to $7.355 \times 10^{-8} \mathrm{~m}^{2} / \mathrm{s}$. These theoretically estimated values of the kinematic viscosities are three orders of magnitude larger than the value of the diffusivity/diffusion coefficients shown in Fig. 11A for IMF2. Therefore, it is unlikely that IMF2 captured the viscosity mode.

A possible explanation of the presence of a second diffusion coefficient associated with IMF2 could be due to the fractal nature of fluctuations. Many researchers acknowledged that "The traditional models for phase separation, nucleation and growth, and spinodal decomposition, are thermodynamic models. These models successfully describe the essential experimental observations in most systems. There is reason to believe, however, that unique, possibly fractal, patterns exist in the earliest stages of phase separation where kinetic growth processes are active." [33] Molecular dynamics simulations [34] reported cluster formation in supercritical Lennard-Jones fluid theoretically explained based on percolation theory [35]. Above $T_{c}$, large clusters form as the density approaches the critical density, following Fisher's droplet model [36]. "The supercritical 
fluctuations show a large and fractal-like structure of the cluster, which has little density dependence" [36]. The existence of a large spatial scale IMF2 above critical temperature could be due to percolating transition loci[37, 38] and the more recent hypothesis of a supercritical mesophase [39, 40]. According to the supercritical mesophase hypothesis, below $T_{c}$, there is a familiar mixing of liquid-gas phases. Above $T_{c}$, "there is a mesophase confined within percolation loci that bound the gas and liquid phases by higher-order discontinuities" [37, 38].

The multiscale analysis allows us to separate the two patterns in the relaxation time of fluctuations and fit them separately with the simplest possible model. Eq. 2 allowed us to extract information about diffusion at different spatial scales. The critical wavenumber $q_{c}$ in Eq. 2 is roughly estimated by the wavenumber at the peak of the relaxation time curves shown in Fig. 7. For wavenumbers larger than the value $q_{c}$ of the critical wavenumber, the approximate fitting function from Eq. 2 becomes $\tau=1 /\left(D q^{2}\right)$, shown with a dashed line and marked with a slope of -2 in Fig. 7. For smaller wavenumbers, the relaxation time's slope is +2 (see Fig. 7). The relaxation time error bars are only shown for the UP region (see Fig. 7A) to avoid cluttering the second panel where the large error bars for IMF3 would cover the data for IMF1, IMF2, and original images.

Because most of the information about the relaxation time of fluctuations is contained in the first IMF1, it masks the contribution of the second IMF. Without first breaking the images into IMFs by using the BEMD technique, we would not have known that there are multiple modes in the original image. Finally, the relaxation time of the third IMF (see solid inverted triangles in Fig. 7) has no structure a ss all wavenumbers.

The diffusion coefficient of the original images (see solid squares in Fig. 8A1) matches our previous results [5]. We show on the same plot both the diffusivity for UP and the effective diffusion coefficient for DOWN results with the origin of time set at the first image of the UP region.

Two new findings are worth noting: (1) for temperatures approaching $T_{c}$ from above, the diffusivity coefficient has a decreasing trend, and (2) on the 
DOWN plateau below $T_{c}$, there is an increasing trend for the effective diffusion

coefficient. The effective diffusion coefficient remains approximately constant around $(2.21 \pm 0.08) \times 10^{-8} \mathrm{~cm}^{2} / \mathrm{s}$ during the first $5 \mathrm{~s}$ of DOWN region and then increases as $(1.01 \pm 0.30) \times 10^{-8}+(1.71 \pm 0.25) \times 10^{-9} \times$ time. The diffusivity (above $T_{c}$ )/effective diffusion coefficient (below $T_{c}$ ) obtained by applying DDM to the first IMF (see solid circles in Fig. 8) closely follows the diffusivity/effective diffusion coefficient values obtained after applying DDM to the original set of images (solid square). At the same time, the diffusivity/effective diffusion coefficient obtained by applying DDM to the IMF2 (see solid triangles in Fig. 8A1) is significantly larger (by one order of magnitude) than that obtained from the original images and is almost constant at around $(4.28 \pm 0.11) \times 10^{-7} \mathrm{~cm}^{2} / \mathrm{s}$.

For the first IMF (solid circles in Fig. 8B), the critical wavenumbers are smaller than those for the original image (solid squares in Fig. 8B1). This is consistent with the relaxation time of the fluctuations plot shown in Fig. 7. Indeed, the relaxation time of the shortest spatial scale of fluctuations, i.e., IMF1, peaks at larger wavenumbers compared to IMF2 (see Fig. 7). Since the soo original image's relaxation time combines the two relaxation times of IMF1 and IMF2, its peak is slightly shifted towards lower wavenumbers compared to the peak of IMF1.

This shifting effect is shown in Fig. 8B1. At the same time, the fact that the critical wavenumbers of the original image are not midway between the critical wavenumbers corresponding to IMF1 and IMF2, respectively, suggests that they may contribute to the total relaxation time with different weights (as seen from Fig. 7).

The diffusivity is made of two parts: a background contribution and a critical part [5]. The most straightforward approach to computing critical contribution is based on the Stokes-Einstein equation [41]:

$$
D_{c}=\frac{R k_{B} T}{6 \pi \eta \xi}
$$

where $R$ is a universal constant close to unity, $k_{B}$ is Boltzmann's constant, $\eta$ is fluid viscosity, and $\xi=\xi_{+} \epsilon^{-\nu}$ is the correlation length. 
Direct measurements of the viscosity of $\mathrm{SF}_{6}$ in the critical region are relatively scarce. One of the first studies done by Henry et al. [42] examined viscosity data reported for other fluids near the critical point (xenon, CO, argon, krypton, nitrogen, oxygen, ethane, methane, and propane) and established a relation for the corresponding states. Combining results from [42] with the low-density $\mathrm{SF}_{6}$ viscosity data $[43,44]$ yields $\eta\left(\rho_{c}, T_{c}\right) \approx 3.47 \times 10^{-5} \mathrm{~Pa} \cdot s$ $[45,46]$. Wu and Webb [46] found that for a reduced temperature range of $1.22 \times 10^{-4} \leq \epsilon \leq 6.90 \times 10^{-2}$, there is no critical anomaly in share viscosity of $\mathrm{SF}_{6}$ and $\eta=\left(425+14.5\left(T_{c}-T\right) \pm 15\right) \times 10^{-7} \mathrm{~Pa} \times s$. A recent review of $\mathrm{SF}_{6}$ data by Guder and Wagnera [47] used the following critical point values $T_{c}=(318.7232 \pm 0.0020) \mathrm{K}, p_{c}=(3.754983 \pm 0.000200) \mathrm{MPa}$, and $\rho_{c}=(742.3 \pm 0.3) \mathrm{kg} / \mathrm{m}^{3}$ (see [48] for $\mathrm{SF}_{6}$ critical region data around $50 \mathrm{mK}$ of $\left.T_{c}\right)$.

Based on our previous results $[5,17]$ we know that the $\xi_{+}=1.8 \times 10^{-10}$ $\mathrm{m}, \xi_{-}=\xi_{+} / 2$, the temperature of the DOWN region is about $47 \mu \mathrm{K}$ below $T_{c}$ based on histogram method [17] and in the range of $15 \mu \mathrm{K}$ to $42 \mu \mathrm{K}$ based on DDM method [5]. As a result, $\xi=\xi_{-} \epsilon^{-\nu}=1.8 \mu \mathrm{m}$ to $3.7 \mu \mathrm{m}$, which places the critical part of the diffusion coefficient in the range $D_{c}=1.5 \times 10^{-8} \mathrm{~cm}^{2} / \mathrm{s}$ to $3.0 \times 10^{-8} \mathrm{~cm}^{2} / \mathrm{s}$. The agreement of the above crude estimation with our experimental findings shown in Fig, 8 is very good compared to the original and IMF1 data. More accurate estimates of the diffusion coefficient can be obtained with more elaborated corrections derived in [49] and [32]. Table VII of [32] summarizes known experimental data on $\mathrm{SF}_{6}$ diffusivity variation with temperature differences $\Delta T=T-T_{c}$ in the range of $198.2 \mathrm{mK}$ down to $1.4 \mathrm{mK}$ above $T_{c}$. We found that the log-log plot of the data (not shown) follows a linear trend with

$$
\log _{10} D=-0.167 \pm 0.03+(0.76 \pm 0.02) * \log _{10} \Delta T
$$

with a reduced $\chi^{2}=0.025$, a coefficient of determination $R^{2}=0.989$, and an adjusted $R^{2}=0.989$. In our experiments, the temperature quench was $0.3 \mathrm{mK}$, and the DOWN region was estimated between $47 \mu \mathrm{K}$ [17] and about $15 \mu \mathrm{K}$ to 
$42 \mu \mathrm{K}$ [5] below $T_{c}$. As a result, extrapolating the above linear trend in log$\log$ coordinates from [32], the diffusion coefficient in our experiments should be in the range $2.1 \times 10^{-8} \mathrm{~cm}^{2} / \mathrm{s}$ to $3.1 \times 10^{-8} \mathrm{~cm}^{2} / \mathrm{s}$. Again, our experimental findings concerning the effective diffusion coefficient shown in Fig. 8 based on this BEMD-DDM technique are in the range consistent with other experiments when compared against the original and IMF1.

\section{Concluding Remarks}

Using the MIR space station's microgravity conditions and the performing thermal and optical environments of the ALICE 2 facility, we have observed the $\mathrm{SF}_{6}$ critical density fluctuations data extremely close to $T_{c}[15,16]$. We previously used the DDM method [5] with a single characteristic time (single exponential) and fitted the ISF of the critical density fluctuations in the homogeneous domain (UP) above the critical point of $\mathrm{SF}_{6}$. The DDM method was extended to the nonhomogeneous domain (DOWN), introducing an effective diffusion coefficient whose physics appeared complex to understand due to the transient nature of the various possible thermal relaxation processes. A closer inspection of the ISF for UP and DOWN regions shows that, although a single exponential covers quite a wide range of delay times, it is not perfect, especially regarding the current theoretical description of the critical phenomena. Therefore we used another tool, named the Bidimensional Empirical Mode Decomposition (BEMD) algorithm, to separate the spatial scales of the physical phenomena that can contribute to light scattering imaging. Although we used the same dataset as in [5], there are fundamental differences in the approach to time scale separation that emerged naturally from the spatial scale separation with BEMD. The most exciting aspect consists of the clear identification at the first order of the IMF, of the unique relaxation time of the well-defined critical density fluctuations that are unambiguously the dominant microscopic physics in the UP region. Although the fundamental molecular mechanism that drives fluctuations at all scales ultimately relies on the same microscopic interaction 

progressively decreases to $\xi_{-}=\xi_{+} / 2$ (its value below $T_{c}$ ). The relaxation time of fluctuations in the second IMF corresponds to the coarser image texture most likely related to small clusters that start appearing below $T_{c}$. Indeed, the critical wavenumber of IMF2 is less than half of the IMF1 (compare solid triangles

potentials among molecules, it does not preclude short spatial scale fluctuations from having a different relaxation time than the large critical scale of the density fluctuations. Therefore, further dynamic investigations using the DDM method at a higher-order IMFs concern only physics, which may have quite large characteristic spatial scales.

Finally, as the BEMD algorithm is data-driven and fully unsupervised, we have here shown that its use is also suitable for the analysis of locally nonlinear and nonstationary data, such as the one obtained from a quick thermal quench that stepped through the critical temperature in our experiment. The BEMD decomposes the spatial frequency components into a set of Intrinsic Mode Function (IMFs). The highest spatial frequency component of each spatial location is in the first IMF, and the second-highest spatial frequency component of each spatial position is in the second IMF, etc. We found that the individual IMFs are described by single-exponential ISF, which provided a natural temporal scale separation. The main focus is on the first two IMFs: the first IMF for the shortest spatial scale is related directly to critical point fluctuations. The second IMF reveals a coarser structure determined by the initial stage of cluster formation and phase separation below $T_{c}$. The relaxation time of fluctuation in the original image (see solid squares in Fig. 7) is captured accurately (more than $90 \%$ cross-correlation) by the first IMF, i.e., the finest spatial scale of fluctuations. This suggests that very close to the critical point, the shortest scale fluctuations dominate and the diffusivity (above $T_{c}$ ) or the effective diffusion coefficient (below $T_{c}$ ), respectively, obtained from the relaxation time of fluctuations in the original image is almost identical with that obtained from the first IMF. It is worth mentioning that the spatial scale that corresponds to the first IMF is of the order of magnitude of the correlation length, i.e., $\xi_{+} \approx 2 \mu \mathrm{m}$ (above $T_{c}$ ) and (IMF2) against solid circles (IMF1) in Fig. 7). As a result, the characteristic 
length of the fluctuations in IMF2 is at least twice the value of IMF1 without any reasonable physical understanding.

A significant improvement in the accuracy of our fitting could be obtained by increasing the resolution and the sampling time of data acquisition in future microgravity experiments. While the current background relaxation time for IMF2 (measured at large wavenumbers) of $0.18 \mathrm{~s}$ is well above the $0.04 \mathrm{~s}$ delay between successive frames, it is less than one frame distance from the $0.15 \mathrm{~s}$ background relaxation time. A faster data acquisition would allow us a more accurate estimation of the diffusivity/diffusion coefficients.

\section{Appendix}

\subsection{Brief review of Differential Dynamic Microscopy (DDM) method}

Cerbino and Trappe [1] and Giavazzi et al. [50] revolutionized the DLS technique by using an ordinary white-light microscope for the study of the wavenumber-dependent dynamics of colloidal dispersions with their new Differential Dynamic Microscopy (DDM) method. The method is described in detail for near-field measurements in the seminal work of Cerbino and Vailati [2]. The DDM uses differences between images separated by a fixed delay time $\delta t$, called fluctuation images $i(\vec{r}, \delta t)$, to extract information regarding the correlation changes' time scale. To this end, the power spectrum $I(\vec{q}, \delta t)$ of the fluctuation images $i(\vec{r}, \delta t)$ maps the real space correlations among particles into wavenumber space $\vec{q}$ (see [5] for the implementation details of the DDM algorithms). Here $q$ is related to the "length scale" $\lambda$ of the correlation among particles through $q=\frac{2 \pi}{\lambda}$. The power spectrum $I(\vec{q}, \delta t)$ is related to the Intermediate Scattering Function (ISF), $G(\vec{q}, \delta t)$, of the system through $[1,50]$ :

$$
I(\vec{q}, \delta t)=A(\vec{q})(1-G(\vec{q}, \delta t))+B(\vec{q})
$$

where $A(\vec{q})$ and $B(\vec{q})$ are determined by the static scattering properties of the sample. The ISF, $G(\vec{q}, \delta t)$, characterizes how quickly a structure is lost over a length scale $\lambda$. Generally, $G(\vec{q}, \delta t)$ monotonically decays to zero over time 
because of dissipative flows. It can be shown that in simple Newtonian fluids the ISF is a Gaussian, i.e. $G(\vec{q}, \delta t)=e^{-\frac{q^{2}}{4}<r^{2}(\delta t)>}$ with the average displacement $<r^{2}(\delta t)>=4 D \delta t$, where $D$ is the diffusion coefficient.

The new method we proposed here is a data-driven multiscale DDM in the sense that the spatial scales are naturally separated based on the image content. To achieve this goal, we used the Empiric Mode Decomposition (EMD) to break the original image into different spatial scales called Intrinsic Mode Function (IMF) and a residual quantity.

We can thus identify individual contributions of different relaxation times of fluctuations at different scales. We focused mainly on the first two IMFs: the first IMF for the shortest spatial scale is related directly to critical point fluctuations (above $T_{c}$ ), and the second IMF reveals a coarser structure that is determined by the initial stage of cluster formation and phase separation (below $\left.T_{c}\right)$.

\subsection{Brief review of the Empirical Mode Decomposition (EMD) method}

The definition of EMD and the related theory were proposed by Huang [51]. Among other advantages over traditional spectral analysis methods, such as Fourier or wavelets, the EMD applies even to nonlinear and nonstationary signals. At the same time, the resultant IMF features are linear and stationary [52]. The EMD has been used, among other research areas, in earthquake analysis $[53,54]$, structural diagnosis $[55,56]$, characterization of nonstationary biological processes [57], mechanical fault diagnosis [58, 59], and ocean waves analysis [60]. The algorithm is as follows. The EMD is a data-driven and adaptive approach to remove oscillations (IMFs) successively through repeated subtraction of the baselines.

1. Let us designate the original signal $x(t)$, with $t>0$ as the original residual time series $r_{0}(t)=x(t)$ and set the iteration index for IMF to $i=1$ (see Fig. $12 \mathrm{~A} 1$ continuous line).

2. Identify all the extrema in $r_{i-1}(t)$ and connect the sequential local maxima $\max _{i-1}(t)$ (respective minima, $\min _{i-1}(t)$ ) using a cubic spline to derive 
the upper. In Fig. 12A1, the maxima envelope is shown with a dashed line and of minima with a dashed-dotted line.

3. Derive the baseline, $m_{i-1}(t)$, by averaging the upper and the lower envelopes (see Fig. 12B1 dashed line), namely:

$$
m_{i-1}(t)=\frac{\max _{i-1}(t)+\min _{i-1}(t)}{2} .
$$

4. Extract the temporary local oscillation $h_{i}(t)=r_{i-1}(t)-m_{i-1}(t)$ (see Fig. 12C1 dashed line). The signal fluctuation $h_{i}(t)$ must represent a pure "oscillation about the mean," i.e., it must satisfy two conditions: (i) in the whole data set, the number of extrema and the number of zero-crossings must either be equal or differ at most by one, and (ii) at any time, the mean value of the envelope of the local maxima and the envelope of the local minima must be zero. An oscillatory function that satisfies these two conditions is called an Intrinsic Mode Function (IMF).

5. If the mean of $h_{i}(t)$ is not zero, repeat steps (2)-(4) on the temporary local oscillation by setting $r_{i-1}(t)=h_{i}(t)$ and iterating until the mean of $h_{i}(t)$ is zero. Then, $h_{i}(t)$ is treated as an IMF, noted as $I M F_{i}(t)$ (see dashed line in Fig. 12C1).

6. Compute the new residue: $r_{i}(t)=r_{i-1}(t)-I M F_{i}(t)$ (see Fig. $12 \mathrm{~A} 2$ continuous line).

7. Increment the IMF index $i$ and return to step (2) to generate series of IMFs and a residue until the residual $r_{i}(t)$ is a constant, or a trend, or has no more than three extrema (see Fig. 12B2-C2). 

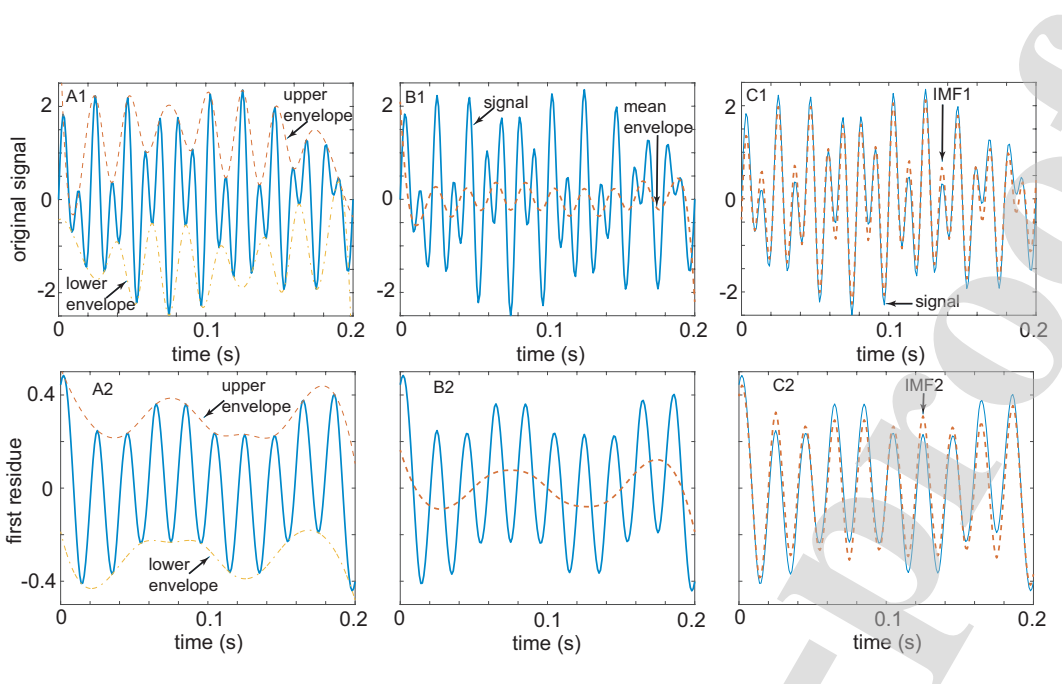

Figure 12: The original signal (continuous line in A1) has a smooth upper envelope (dashed line in A1) and lower envelope (dashed-dotted line in A1). The two envelopes from A1 determine the mean envelope in B1 (dashed line). By subtracting the mean envelope of B1 from the signal, one obtains the first IMF in $\mathrm{C} 1$ (dashed line). The difference between the signal (continuous line in C1) and the first IMF (dashed line in C1) gives the first residue in A2 (continuous line). The process depicted in panels A1-C1 is repeated with the signal shown in A2 until one obtains the second IMF in $\mathrm{C} 2$. This one-dimensional signal was generated from an analytic function to demonstrate the use of the EMD technique. The BEMD follows the same steps, except the envelopes are two-dimensional surfaces.

The iterative sifting process of the EMD technique can be continued until either the residue or the intrinsic mode becomes less than a predetermined small number, or the residue becomes nonoscillatory. For a sifting process that identifies $n$ IMFs, the original signal is simply

$$
x(t)=\sum_{i=1}^{n} I M F_{i}+r_{n},
$$

where $r_{n}$ represents the nonoscillatory trend of the signal. Usually, in the case of bidimensional signals (images), the last residue represents the prevailing trend in images (background) due to nonuniform illumination and could be used to correct the images.

Since its inception, many significant improvements to EMD have been made, e.g., extra Gaussian noise was added to data in Ensemble Empirical Mode De- 
composition (EEMD) to alleviate the aliasing phenomenon [61]. Eliminating the residual noise introduced in EEMD was achieved recently by Complementary Ensemble Empirical Mode Decomposition (CEEMD) theory [62]. The EMD was expanded to Bidimensional Empirical Mode Decomposition (BEMD) [63] and applied to remote sensing [64]. The BEMD is a data-driven method, and it requires no pre-determined filter or wavelet functions [65]. The BEMD has also been applied to texture extraction and image filtering [66], finding the gold mineral deposition [67] and tin-copper polymetallic ore fields [68] from gravity anomalies captured by satellite images, image denoising [69, 70], content-based image retrieval [71], and fusion of multispectral and remote sensing [72, 73]. There are various implementations of the BEMD method, depending on the surface interpolation algorithm used. For one-dimensional signals (time series), one of the standards methods for connecting minima/maxima is cubic spline interpolation, see, for example, the $e m d()$ standard Matlab function. For twodimensional signals (images), there are multiple possible ways of constructing the interpolation surface that connects all minima (lower envelope) and maxima (upper envelope), respectively. To extract the two-dimensional IMFs during the sifting process ones could use radial basis function [74] or multigrid beta-splines [75] to construct the two-dimensional lower and upper envelopes, respectively. Others treated multidimensional data as a collection of one-dimensional signals [76]. According to such an approach, two-dimensional spatial data or images $I(x, y)$ are a collection of one-dimensional series in both $\mathrm{x}$ - and $\mathrm{y}$-directions. As a result, each of the one-dimensional slices is decomposed with the EMD algorithm by following the seven steps described above. Subsequently, the slice of a similar scale is reconstructed to produce a two-dimensional pseudo-IMF-like component [76].

\section{Acknowledgments}

A.O acknowledges a mini-Research and Education Awards Project grant from NASA South Carolina Space Grant/EPSCoR. Y.G., C.L., and D.B. ac- 
knowledge a research grant from Centre National d'Études Spatiales (CNES)

\section{References}

[1] R. Cerbino, V. Trappe, Differential Dynamic Microscopy: Probing Wave Vector Dependent Dynamics with a Microscope, Physical Review Letters 100 (2008) 188102. doi:10.1103/PhysRevLett.100.188102.

[2] R. Cerbino, A. Vailati, Near-field scattering techniques: Novel instrumentation and results from time and spatially resolved investigations of soft matter systems, Current Opinion in Colloid and Interface Science 14 (6) (2009) 416 - 425. doi:10.1016/j.cocis.2009.07.003.

[3] F. Croccolo, J. M. Ortiz de Zárate, J. V. Sengers, Non-local fluctuation phenomena in liquids, The European Physical Journal E 39 (12) (2016) 125. doi:10.1140/epje/i2016-16125-3.

[4] A. Vailati, R. Cerbino, S. Mazzoni, C. Takacs, D. Cannell, M. Giglio, Fractal fronts of diffusion in microgravity, Nature Communications 2 (290) (2011) 1-5. doi:10.1038/ncomms 1290.

[5] A. Oprisan, S. Oprisan, B. Bayley, J. Hegseth, Y. Garrabos, C. LecoutreChabot, D. Beysens, Dynamic structure factor of density fluctuations from direct imaging very near (both above and below) the critical point of $\mathrm{SF}_{6}$, Physical Review E 86 (2012) 061501. doi:10.1103/PhysRevE.86.061501.

[6] O. Bondarchuk, D. B. Dougherty, M. Degawa, E. D. Williams, M. Constantin, C. Dasgupta, S. Das Sarma, Correlation time for step structural fluctuations, Physical Review B 71 (2005) 045426. doi:10.1103/ PhysRevB. 71.045426.

[7] F. Croccolo, Dynamics of Non Equilibrium Fluctuations in Free Diffusion, Thesis (2006). 
[8] A. Vailati, M. Giglio, Nonequilibrium fluctuations in time-dependent diffusion processes, Physical Review E 58 (1998) 4361-4371. doi:10.1103/ PhysRevE.58.4361.

[9] F. Croccolo, C. Giraudet, H. Bataller, R. Cerbino, A. Vailati, Shadowgraph Analysis of Non-equilibrium Fluctuations for Measuring Transport Properties in Microgravity in the GRADFLEX Experiment, Microgravity Science and Technology 28 (4) (2016) 467-475. doi:10.1007/s12217-016-9501-1.

[10] F. Giavazzi, A. Fornasieri, A. Vailati, R. Cerbino, Equilibrium and nonequilibrium concentration fluctuations in a critical binary mixture, The European Physical Journal E 39 (10) (2016) 103. doi:10.1140/epje/ i2016-16103-9.

[11] F. Giavazzi, G. Savorana, A. Vailati, R. Cerbino, Structure and dynamics of concentration fluctuations in a non-equilibrium dense colloidal suspension, Soft Matter 12 (2016) 6588-6600. doi:10.1039/C6SM00935B.

[12] F. Croccolo, D. Brogioli, A. Vailati, M. Giglio, D. Cannell, Use of dynamic schlieren interferometry to study fluctuations during free diffusion, Applied Optics 45 (10) (2006) 2166-2173. doi:10.1364/A0.45.002166.

[13] H. Bataller, C. Giraudet, F. Croccolo, J. Maria Ortiz de Zarate, Analysis of Non-Equilibrium Fluctuations in a Ternary Liquid Mixture, Microgravity Science and Technology 28 (2016). doi:10.1007/s12217-016-9517-6.

730 [14] J. Ortiz de Zarate, C. Giraudet, H. Bataller, F. Croccolo, Non-equilibrium fluctuations induced by the Soret effect in a ternary mixture, The European Physical Journal E: Soft Matter 37 (2014) 34. doi:10.1140/epje/ i2014-14077-2.

[15] R. Marcout, J. Zwilling, J. Laherrere, Y. Garrabos, B. D., ALICE 2, an advanced facility for the analysis of fluids close to their critical point in microgravity, in: 45th Congress of the International Astronautical Federation, Jerusalem, Israel, International Astronautical Federation, 1994. 
[16] C. Lecoutre, Y. Garrabos, E. Georgin, F. Palencia, D. Beysens, Turbidity data of weightless $\mathrm{SF}_{6}$ near its liquid-gas critical point, International Journal of Thermophysics 30 (3) (2009) 810-832. doi:10.1007/ s10765-009-0582-6.

[17] A. Oprisan, S. A. Oprisan, J. J. Hegseth, Y. Garrabos, C. Lecoutre-Chabot, D. Beysens, Universality in early-stage growth of phase-separating domains near the critical point, Physical Review E 77 (5 Pt 1) (2008) 051118.

[18] A. Durieux, B. Martin, D. Laubier, Declic: design, integration and testing of a multi configurable instrument using optical diagnostics to study directional solidification and critical fluids, in: E. Armandillo, J. Costeraste, N. Karafolas (Eds.), International Conference on Space Optics - ICSO 2006, Vol. 10567, International Society for Optics and Photonics, SPIE, 2017, pp. 951 - 957. doi:10.1117/12.2308146.

[19] Y. Garrabos, C. Lecoutre, S. Marre, R. Guillaument, D. Beysens, I. Hahn, Crossover equation of state models applied to the critical behavior of xenon, J. Stat. Phys. 158 (2015) 1379-1412. doi : 10.1007/s10955-014-1157-x.

[20] Y. Garrabos, C. Lecoutre, S. Marre, I. Hahn, Critical crossover functions for simple fluids: Towards the crossover modelling uniqueness, J. Stat. Phys. 165 (2016) 471-506. doi:10.1007/s10955-016-1627-4.

[21] Y. Garrabos, C. Lecoutre, S. Marre, D. Beysens, I. Hahn, Liquid-vapor rectilinear diameter revisited, Phys. Rev. E 97 (2018) 020101. doi:10. 1103/PhysRevE.97.020101.

[22] C. Lecoutre, Y. Garrabos, D. Beysens, V. Nikolayev, I. Hahn, Boiling phenomena in near-critical sf6 observed in weightlessness, Acta Astronautica 100 (2014) 22 - 29. doi:https://doi.org/10.1016/j.actaastro. 2014. 03.012 .

[23] C. Lecoutre, R. Guillaument, S. Marre, Y. Garrabos, D. Beysens, I. Hahn, 
Weightless experiments to probe universality of fluid critical behavior, Phys. Rev. E. 91 (6) (2015) 06010. doi:10.1103/PhysRevE.91.060101.

[24] F. Mota, N. Bergeon, D. Tourret, A. Karma, R. Trivedi, B. Billia, Initial transient behavior in directional solidification of a bulk transparent model alloy in a cylinder, Acta Materialia 85 (2015) 362 - 377. doi:https://doi.org/10.1016/j.actamat.2014.11.024.

URL http://www.sciencedirect.com/science/article/pii/ S1359645414008775

[25] V. Nikolayev, Y. Garrabos, C. Lecoutre, T. Charignon, D. Hitz, D. Chatain, R. Guillaument, S. Marre, D. Beysens, Boiling crisis dynamics: Low gravity experiments at high pressure, Microgravity Science and Technology 27 (4) (2015) 253-260. doi:10.1007/s12217-015-9447-8.

URL https://doi.org/10.1007/s12217-015-9447-8

[26] F. Croccolo, D. Brogioli, A. Vailati, M. Giglio, D. Cannell, Nondiffusive decay of gradient-driven fluctuations in a free-diffusion process, Physical Review E 76 (4) (2007) 041112. doi:10.1103/PhysRevE.76.041112.

[27] A. Onuki, Phase transition dynamics, Cambridge University Press, Cambridge, UK, 2002.

[28] K. Kawasaki, Kinetic equations and time correlation functions of critical fluctuations, Annals of Physics 61 (1) (1970) 1-56. doi:10.1016/ 0003-4916 (70) 90375-1.

[29] J. Oh, J. Ortiz de Zárate, J. Sengers, G. Ahlers, Dynamics of fluctuations in a fluid below the onset of Rayleigh-Bénard convection, Physical Review E 69 (2004) 021106. doi:10.1103/PhysRevE.69.021106.

[30] H. Tanaka, T. Nishi, Direct determination of the probability distribution function of concentration in polymer mixtures undergoing phase separation, Physical Review Letters 59 (1987) 692-695. doi:10.1103/PhysRevLett. 59.692 . 
[31] M. Moldover, J. Sengers, R. Gammon, R. Hocken, Gravity effects in fluids near the gas-liquid critical point, Review of Modern Physics 51 (1) (1979) 79-99. doi:10.1103/RevModPhys.51.79.

[32] R. A. Wilkinson, G. A. Zimmerli, H. Hao, M. Moldover, R. Berg, W. Johnson, R. Ferrell, R. Gammon, Equilibration near the liquid-vapor critical point in microgravity, Physical Review E 57 (1) (1998) 436-448. doi:10.1103/PhysRevE.57.436.

[33] D. W. Schaefer, B. C. Bunker, J. P. Wilcoxon, J. S. Rowlinson, R. C. Ball, D. J. Tildesley, M. Fleischmann, Fractals and phase separation, Proceedings of the Royal Society of London. A. Mathematical and Physical Sciences 423 (1864) (1989) 35-53. doi:10.1098/rspa.1989.0040.

[34] D. Heyes, Cluster analysis and continuum percolation of 3d square-well phases mc and py solutions, Molecular Physics 69 (3) (1990) 559-569. doi: $10.1080 / 00268979000100401$.

[35] D. Stauffer, A. Aharony, Introduction To Percolation Theory, Taylor and Francis, 1994.

[36] N. Yoshii, S. Okazaki, A large-scale and long-time molecular dynamics study of supercritical lennard-jones fluid. an analysis of high temperature clusters, The Journal of Chemical Physics 107 (6) (1997) 2020-2033. doi: $10.1063 / 1.474553$.

[37] L. V. Woodcock, Observations of a thermodynamic liquid-gas critical coexistence line and supercritical fluid phase bounds from percolation transition loci, Fluid Phase Equilibria 351 (2013) 25 - 33, special Issue covering the Eighteenth Symposium on Thermophysical Properties. doi:10.1016/j.fluid.2012.08.029.

[38] L. Woodcock, Thermodynamic fluid equations-of-state, Computational Methods in Science and Technology 22 (3) (2017) 22. doi:10.3390/ e20010022. 
[39] H. Magnier, R. Curtis, L. Woodcock, Nature of the supercritical mesophase, Natural Science 6 (2014) 797-807. doi :10.4236/ns . 2014.610078.

[40] L. Woodcock, Percolation transitions and fluid state boundaries, Entropy 20 (1) (2018) 281-294. doi:10.12921/cmst.2016.0000070.

825 [41] S. Will, A. Leipertz, Mutual diffusion coefficient and dynamic viscosity near the critical consolute point probed by dynamic light scattering, International Journal of Thermophysics 20 (3) (1999) 791-803. doi:10.1023/A : 1022670816359.

[42] D. L. Henry, H. L. Swinney, H. Z. Cummins, Rayleigh Linewidth in Xenon Near the Critical Point, Physical Review Letters 25 (1970) 1170-1173. doi : 10.1103/PhysRevLett.25.1170.

[43] J. C. McCoubrey, N. M. Singh, Intermolecular forces in quasi-spherical molecules, Transactions of Faraday Society 53 (1957) 877-883. doi:10. 1039/TF9575300877.

835 [44] J. C. McCoubrey, N. M. Singh, Intermolecular forces in quasi-spherical molecules. Part 2, Transactions of Faraday Society 55 (1959) 1826-1830. doi:10.1039/TF9595501826.

[45] T. Lim, H. Swinney, K. Langley, T. Kachnowski, Rayleigh Linewidth in SF $_{6}$ Near the Critical Point, Physical Review Letters 27 (1971) 1776-1780. doi:10.1103/PhysRevLett.27.1776.

[46] E. S. Wu, W. W. Webb, Critical Liquid-Vapor Interface in $\mathrm{SF}_{6}$. II. Thermal Excitations, Surface Tension, and Viscosity, Physical Review A 8 (1973) 2077-2084. doi:10.1103/PhysRevA.8.2077.

[47] C. Guder, W. Wagnera, A Reference Equation of State for the Thermodynamic Properties of Sulfur Hexafluoride $\left(\mathrm{SF}_{6}\right)$ for Temperatures from the Melting Line to 625k and Pressures up to 150mpa, Journal of Physical and Chemical Reference Data 38 (2009) 33-94. doi:10.1063/1.3037344. 
[48] N. Kurzeja, T. Tielkes, W. Wagner, The Nearly Classical Behavior of a Pure Fluid on the Critical Isochore Very Near the Critical Point Under the Influence of Gravity, International Journal of Thermophysics 20 (2) (1999) 531-561. doi:10.1023/A:1022657121329.

[49] J. Luettmer-Strathmann, J. Sengers, G. Olchowy, Non-asymptotic critical behavior of the transport properties of fluids., Journal of Chemical Physics 103 (17) (1995) 7482-7502.

${ }_{855}$ [50] F. Giavazzi, D. Brogioli, V. Trappe, T. Bellini, R. Cerbino, Scattering information obtained by optical microscopy: Differential dynamic microscopy and beyond, Physical Review E 80 (2009) 031403. doi:10.1103/PhysRevE. 80.031403.

[51] N. Huang, Z. Shen, S. Long, M. Wu, H. Shih, Q. Zheng, N.-C. Yen, C. Tung, H. Liu, The empirical mode decomposition and the hilbert spectrum for nonlinear and non-stationary time series analysis, Proceedings of the Royal Society of London. Series A: Mathematical, Physical and Engineering Sciences 454 (1971) (1998) 903-995. doi:10.1098/rspa.1998.0193.

[52] S. Huang, Z. Liu, Z. Liu, L. Wang, Sar Image Change Detection Algorithm Based on Different Empirical Mode Decomposition, Journal of Computer and Communications 5 (2017) 9-20. doi:10.4236/jcc.2017.58002.

[53] S. Raghukanth, S. Sangeetha, Empirical mode decomposition of earthquake accelerograms, Advances in Adaptive Data Analysis 04 (04) (2012) 1250022. doi:10.1142/S1793536912500227.

[54] J.-Y. Huang, K.-L. Wen, X.-J. Li, J.-J. Xie, C.-T. Chen, S.-C. Su, Coseismic Deformation Time History Calculated from Acceleration Records Using an EMD-Derived Baseline Correction Scheme: A New Approach Validated for the 2011 Tohoku Earthquake, Bulletin of the Seismological Society of America 103 (2B) (2013) 1321-1335. doi:doi.org/10.1785/0120120278. 
[55] A. Garcia-Perez, J. Amezquita-Sanchez, A. Dominguez-Gonzalez, R. Sedaghati, R. Osornio-Rios, R. Romero-Troncoso, Fused empirical mode decomposition and wavelets for locating combined damage in a truss-type structure through vibration analysis, Journal of Zhejiang University SCIENCE A 14 (9) (2013) 615-630.

[56] A. Moreno-Gomez, J. Amezquita-Sanchez, M. Valtierra-Rodriguez, C. Perez-Ramirez, A. Dominguez-Gonzalez, O. Chavez-Alegria, EmdShannon Entropy-Based Methodology to Detect Incipient Damages in a Truss Structure, Applied Sciences 8 (11) (2018). doi:10.3390/ app8112068.

[57] W. Huang, Z. Shen, N. Huang, Y. Fung, Use of intrinsic modes in biology: Examples of indicial response of pulmonary blood pressure to step hypoxia, Proceedings of the National Academy of Sciences 95 (22) (1998) 1276612771. doi:10.1073/pnas.95.22.12766.

[58] J. Zheng, J. Cheng, Y. Yang, Generalized empirical mode decomposition and its applications to rolling element bearing fault diagnosis, Mechanical Systems and Signal Processing 40 (1) (2013) 136 - 153. doi:10.1016/j. ymssp. 2013.04.005.

[59] J. Zheng, H. Pan, S. Yang, J. Cheng, Generalized composite multiscale permutation entropy and laplacian score based rolling bearing fault diagnosis, Mechanical Systems and Signal Processing 99 (2018) 229 - 243. doi:10.1016/j.ymssp. 2017.06.011.

[60] H. Song, H. Bai, L. Pinheiro, C. Dong, C.-Y. Huang, B. Liu, Analysis of ocean internal waves imaged by multichannel reflection seismics, using ensemble empirical mode decomposition, Journal of Geophysical Engineering 9 (2012) 302-311. doi:10.1088/1742-2132/9/3/302.

[61] Z. WU, N. Huang, X. Chen, The Multi-dimensional Ensemble Empirical Mode Decomposition Method, Advances in Adaptive Data Analysis 01 (03) (2009) 339-372. doi:10.1142/S1793536909000187. 
[62] J.-R. Yeh, J.-S. Shieh, N. Huang, Complementary ensemble empirical mode decomposition: A novel noise enhanced data analsis method, Advances in Adaptive Data Analysis 02 (02) (2010) 135-156. doi:10.1142/ S1793536910000422.

[63] G. Rilling, P. Flandrin, P. Goncalves, J. M. Lilly, Bivariate empirical mode decomposition, IEEE Signal Processing Letters 14 (12) (2007) 936-939. doi:10.1109/LSP.2007.904710.

[64] Z. He, Q. Wang, Y. Shen, J. Jin, Y. Wang, Multivariate Gray ModelBased BEMD for Hyperspectral Image Classification, IEEE Transactions on Instrumentation and Measurement 62 (5) (2013) 889-904. doi:10. 1109/TIM. 2013. 2246917.

[65] A. Saha, M. Pukhrambam, S. Pradhan, Facial image analysis for expression recognition by bidimensional empirical mode decomposition, Indian Journal of Science and Technology 9 (31) (2016) 1-9. doi:10.17485/ijst/ 2016/v9i31/90495.

[66] C. Jean, Y. Bouaoune, D. Eric, N. Oumar, P. Bunel, Image analysis by bidimensional empirical mode decomposition, Image Vision and Computation 21 (2003) 1019-1026. doi:10.1016/S0262-8856(03) 00094-5.

[67] J. Huang, B. Zhao, Y. Chen, P. Zhao, Bidimensional empirical mode decomposition (BEMD) for extraction of gravity anomalies associated with gold mineralization in the tongshi gold field, western shandong uplifted block, eastern china, Computers and Geosciences 36 (7) (2010) 987 - 995. doi:10.1016/j.cageo.2009.12.007.

[68] Y. Chen, L. Zhang, B. Zhao, Application of Bi-dimensional empirical mode decomposition (BEMD) modeling for extracting gravity anomaly indicating the ore-controlling geological architectures and granites in the gejiu tincopper polymetallic ore field, southwestern china, Ore Geology Reviews 88 (2017) $832-840$. doi:10.1016/j.oregeorev.2016.06.031. 
[69] F. Ben Arfia, A. Sabri, M. Ben Messaoud, M. Abid, The bidimensional empirical mode decomposition with 2D-DWT for gaussian image denoising, in: 2011 17th International Conference on Digital Signal Processing (DSP), 2011, pp. 1-5. doi:10.1109/ICDSP. 2011 .6004908.

[70] D. Liu, X. Chen, Image denoising based on improved bidimensional empirical mode decomposition thresholding technology, Multimedia Tools and Applications 78 (6) (2019) 7381-7417. doi:10.1007/s11042-018-6503-6.

[71] P. Alvanitopoulos, A. Moroi, G. Bagropoulos, K. Dundon, Content Based Image Retrieval and Its Application to Product Recognition, in: R. Chbeir, Y. Manolopoulos, I. Maglogiannis, R. Alhajj (Eds.), Artificial Intelligence Applications and Innovations, Springer International Publishing, Cham, 2015, pp. 3-18.

[72] W. Dong, X. Li, X. Lin, Z. Li, A Bidimensional Empirical Mode Decomposition Method for Fusion of Multispectral and Panchromatic Remote Sensing Images, Remote Sensing 6 (2014) 8446-8467. doi:10.3390/rs6098446.

[73] L. Guang, L. Li, H. Gong, Q. Jin, X. Li, R. Song, Y. Chen, Y. Chen, C. He, Y. Huang, Y. Yao, Multisource Remote Sensing Imagery Fusion Scheme Based on Bidimensional Empirical Mode Decomposition (BEMD) and Its Application to the Extraction of Bamboo Forest, Remote Sensing 9 (2016) 19. doi:10.3390/rs9010019.

[74] J. Nunes, Y. Bouaoune, E. Delechelle, O. Niang, P. Bunel, Image analysis by bidimensional empirical mode decomposition, Image and Vision Computing 21 (12) (2003) 1019 - 1026. doi:10.1016/S0262-8856(03) 00094-5.

[75] J. C. Nunes, S. Guyot, E. Deléchelle, Texture analysis based on local analysis of the Bidimensional Empirical Mode Decomposition, Machine Vision and Applications 16 (3) (2005) 177-188. doi:10.1007/ s00138-004-0170-5. 
960 [76] Z. Wu, N. Huang, Ensemble empirical mode decomposition: A noise-assited data analysis method, Advances in Adaptive Data Analysis 01 (01) (2009) 1-41. doi:10.1142/S1793536909000047. 